\title{
Elementary Abelian Covers of Graphs
}

\author{
ALEKSANDER MALNIČ* \\ DRAGAN MARUŠIČ* \\ PRIMOŽ POTOČNIK ${ }^{\dagger}$ \\ dragan.marusic@uni-lj.si \\ IMFM, Oddelek za matematiko, Univerza v Ljubljani, Jadranska 19, 1111 Ljubljana, Slovenija
}

Received November 5, 2001; Revised July 24, 2003; Accepted August 7, 2003

Abstract. Let $\mathcal{C}_{G}(X)$ be the set of all (equivalence classes of) regular covering projections of a given connected graph $X$ along which a given group $G \leq$ Aut $X$ of automorphisms lifts. There is a natural lattice structure on $\mathcal{C}_{G}(X)$, where $\wp_{1} \leq \wp_{2}$ whenever $\wp_{2}$ factors through $\wp_{1}$. The sublattice $\mathcal{C}_{G}(\wp)$ of coverings which are below a given covering $\wp: \tilde{X} \rightarrow X$ naturally corresponds to a lattice $\mathcal{N}_{G}(\wp)$ of certain subgroups of the group of covering transformations. In order to study this correspondence, some general theorems regarding morphisms and decomposition of regular covering projections are proved. All theorems are stated and proved combinatorially in terms of voltage assignments, in order to facilitate computation in concrete applications.

For a given prime $p$, let $\mathcal{C}_{G}^{p}(X) \leq \mathcal{C}_{G}(X)$ denote the sublattice of all regular covering projections with an elementary abelian $p$-group of covering transformations. There is an algorithm which explicitly constructs $\mathcal{C}_{G}^{p}(X)$ in the sense that, for each member of $\mathcal{C}_{G}^{p}(X)$, a concrete voltage assignment on $X$ which determines this covering up to equivalence, is generated. The algorithm uses the well known algebraic tools for finding invariant subspaces of a given linear representation of a group. To illustrate the method two nontrival examples are included.

Keywords: covering projection, lifting automorphisms, Cayley voltages, homological covering, invariant subspace, lattice, arc-transitive graph, semisymmetric graph, dipole, Heawood graph

\section{Introduction}

Graphs admitting an imprimitive action of a subgroup of automorphisms arise naturally out of their quotients. In attempting to classify graphs with interesting symmetry properties it is therefore natural to first consider the 'primitive' graphs (which is almost exclusively based on the classification of finite simple groups) and then try to explicitly construct the 'imprimitive' ones. In many instances the 'imprimitive' graphs arise as derived graphs along regular covering projections. Djoković [5] showed that the correspondence between symmetry properties of graphs and symmetry properties of their regular covers is related to the problem of lifting automorphisms.

So the main question addressed in this paper is the following one. For a given connected graph $X$ and a subgroup $G \leq$ Aut $X$ of its automorphisms, find the set $\mathcal{C}_{G}(X)$ of equivalence classes of connected regular $G$-admissible covering projections $\wp: \tilde{X} \rightarrow X$, that is, covering projections along which $G$ lifts. The answer is well understood at some very general 
level in view of the fact that the problem of lifting automorphisms was solved in algebraic topology decades ago. However, finding the required covering projections explicitly is algorithmically almost intractable - this largely depends also on how the term 'explicitly' is to be understood. As each covering projection of graphs can be, up to equivalence, reconstructed in terms of voltages [11], our understanding of 'explicitly' is to display concrete voltage assignments on the graph $X$.

Consider the natural partial ordering $\leq$ in the set $\mathcal{C}_{G}(X)$, where $\wp_{1} \leq \wp_{2}$ whenever $\wp_{2}$ factors through $\wp_{1}[24,25]$. Let $\wp: \tilde{X} \rightarrow X$ be a $G$-admissible regular covering projection, and let $\tilde{G} \leq$ Aut $\tilde{X}$ be the lift of $G$. There is a lattice anti-isomorphism $\Psi_{(G, \wp)}: \mathcal{N}_{G}(\wp) \rightarrow$ $\mathcal{C}_{G}(\wp)$ between the set of regular covering projections $\mathcal{C}_{G}(\wp) \subseteq \mathcal{C}_{G}(X)$ which are below $\wp$ in the ordering, and the set $\mathcal{N}_{G}(\wp)$ of normal subgroups of $\tilde{G} \leq$ Aut $\tilde{X}$ which are contained in the group of covering transformations $\mathrm{CT}(\wp)$. So by finding $\mathcal{N}_{G}(\wp)$ one can determine $\mathcal{C}_{G}(\wp)$ and moreover, $\mathcal{C}_{G}(X)$, since $\mathcal{C}_{G}(X)=\mathcal{C}_{G}\left(\wp_{U}\right)$, where $\wp_{U}$ is the universal covering projection of an appropriate (infinite) tree onto $X$.

A regular covering projection is $p$-elementary abelian if its group of covering transformations is an elementary abelian $p$-group. In this paper we show how to explicitly construct, for a given prime $p$, the sublattice $\mathcal{C}_{G}^{p}(X) \subseteq \mathcal{C}_{G}(X)$ of $p$-elementary abelian $G$-admissible regular covering projections. Namely, the lattice $\mathcal{C}_{G}^{p}(X)$ has a unique maximal element, the $p$-homological covering $\wp_{\hbar}: X_{\hbar}^{p} \rightarrow X$, which is characterized by having $\mathrm{CT}\left(\wp_{\hbar}\right)$ isomorphic to the first homology group $H_{1}\left(X ; \mathbb{Z}_{p}\right)$. Since $\wp_{\hbar}$ is maximal, we have $\mathcal{C}_{G}^{p}(X)=\mathcal{C}_{G}\left(\wp_{\hbar}\right)$. Moreover, finding $\mathcal{N}_{G}\left(\wp_{\hbar}\right)$ is now reduced to finding all invariant subspaces for the linear representation of $G$ on $H_{1}\left(X ; \mathbb{Z}_{p}\right)$. Once these subspaces are found, the elements of $\mathcal{C}_{G}^{p}(X)$ can be explicitly reconstructed in terms of concrete voltage assignments on $X$.

The paper is organized as follows. In Section 2 we review some preliminary concepts about graphs and (combinatorial) regular covering projections in terms of voltages. In order to have a broader range of applications we allow graphs to have semiedges. (Note that the theory of topological coverings and in particular, standard theorems about lifting automorphisms as known in topology do not apply directly in this case since a walk of length 1 traversing a semiedge should be considered as homotopically nontrivial.) In Section 3 we prove a general theorem about the existence of certain morphisms between regular covering projections to be used repeatedly later on. In Section 4 we revise some results of Venkatesh $[24,25]$ about ordering regular $G$-admissible covering projections. Our treatement is combinatorial in terms of voltages and hence differs from the one given in [24,25]. Moreover, we show how to construct the covering projection $\Psi_{(G, \wp)}(K)\left(K \in \mathcal{N}_{G}(\wp)\right)$ in terms of voltages, see Proposition 4.1(b). In Section 5 we specialize the general considerations of previous sections to abelian regular covering projections. This is then used in Section 6, where elementary abelian coverings are treated in detail. The central result, Theorem 6.2, upgrades Proposition 4.1(b); in particular, we show how to construct $\Psi_{(G, \wp)}(K)$ $\left(K \in \mathcal{N}_{G}(\wp)\right)$ explicitly if the voltage group is elementary abelian. From this, an algorithm which determines all $G$-admissible $p$-elementary abelian regular covering projections follows (see Corollary 6.5). The method reduces to finding all invariant subspaces of a linear representation of a group on the first homology group $H_{1}\left(X ; \mathbb{Z}_{p}\right)$. It is generally known that such invariant subspaces can be found by considering the factorization of the respective minimal polynomials. The method generalizes Širáňs treatement of prime-cyclic covering 
projections [22]. Finally, in Section 7 we provide two fairly detailed concrete examples, namely, the elementary abelian regular covering projections of prime-dipoles and of the Heawood graph.

For additional references on graph coverings related to the topics of this article we refer the reader to $[1,11,12]$.

\section{Preliminaries}

\subsection{Graphs}

For the purpose of this article, a graph is an ordered 4-tuple $X=(D, V$; beg, inv $)$ where $D$ and $V \neq \varnothing$ are disjoint finite sets of darts and vertices, respectively, beg : $D \rightarrow V$ is a mapping which assigns to each dart $x$ its initial vertex beg $x$, and inv : $D \rightarrow D$ is an involution which interchanges every dart $x$ and its inverse dart $x^{-1}=\operatorname{inv} x$. If not explicitly given, the four defining parameters of a graph $X$ are denoted by $D_{X}, V_{X}$, beg and inv $_{X}$, respectively. The terminal vertex end $x$ of a dart $x$ is the initial vertex of inv $x$. The orbits of inv are called edges. An edge is called a semiedge if inv $x=x$, a loop if inv $x \neq x$ while end $x=\operatorname{beg} x$, and is called a link otherwise.

Walks are defined as sequences of darts where for any two consecutive darts $x, y$ we have end $x=\operatorname{beg} y$. By recursively deleting all consecutive occurrences of a dart and its inverse in a given walk we obtain a reduced walk which we call the reduction. Two walks with the same reduction are called homotopic. The naturally induced operation on the set of all reduced $b$-based closed walks defines the fundamental group $\pi(X, b)$ at the base vertex $b$. Note that the concept of homotopy does not exactly correspond to homotopy in the corresponding 1-CW complex associated with a graph. This is due to the fact that a walk of length 1 traversing a semiedge is homotopically nontrivial. Thus, the fundamental group is not a free group in general. (Yet a minimal generating set of $\pi(X, b)$ can be obtained in the standard way employing a spanning tree.) Consequently, the first homology group $H_{1}(X)$, obtained by abelianizing $\pi(X, b)$, is not necessarily a free $\mathbb{Z}$-module. Namely, let $r_{e}+r_{s}$ be the minimal number of generators of $\pi(X, b)$, where $r_{s}$ is the number of semiedges and $r_{e}$ is the number of cotree loops and links relative to some spanning tree. Then $H_{1}(X) \cong \mathbb{Z}^{r_{e}} \times \mathbb{Z}_{2}^{r_{s}}$. The first homology group $H_{1}\left(X ; \mathbb{Z}_{p}\right) \cong H_{1}(X) / p H_{1}(X)$ with $\mathbb{Z}_{p}$ as the coefficient ring can be considered as a vector space over the field $\mathbb{Z}_{p}$. Observe that

$$
H_{1}\left(X ; \mathbb{Z}_{p}\right) \cong\left\{\begin{array}{ll}
\mathbb{Z}_{p}^{r_{e}+r_{s}} & p=2 \\
\mathbb{Z}_{p}^{r_{e}} & p \geq 3
\end{array} .\right.
$$

A morphism of graphs $f: X \rightarrow X^{\prime}$ is a function $f: V_{X} \cup D_{X} \rightarrow V_{X^{\prime}} \cup D_{X^{\prime}}$ such that $f V_{X} \subseteq V_{X^{\prime}}, f D_{X} \subseteq D_{X^{\prime}}$, and $f \operatorname{beg}_{X}=\operatorname{beg}_{X^{\prime}} f$ and $f \operatorname{inv}_{X}=\operatorname{inv}_{X^{\prime}} f$. The product of graph morphisms is defined as composition of functions. Concepts such as mono-, epi-, iso- and automorphisms are self-explanatory. An important special case of morphisms arises in the context of group actions on graphs. Namely, if $N \leq$ Aut $X$ is a subgroup of automorphisms let $D_{N}=\{[x] \mid x \in D\}$ and $V_{N}=\{[v] \mid v \in V\}$ denote the sets of $N$-orbits on darts and vertices of $X$, respectively, and let beg $\operatorname{sen}_{N}[x]=[\operatorname{beg} x] \operatorname{and~inv}_{N}[x]=[\operatorname{inv} x]$. 
This defines a graph $X_{N}=\left(D_{N}, V_{N} ;\right.$ beg $_{N}$, inv $\left._{N}\right)$ together with a natural epimorphism $p_{N}: X \rightarrow X_{N}$ called the quotient projection relative to $N$.

\subsection{Covering projections}

A graph epimorphism $\wp: \tilde{X} \rightarrow X$ of connected graphs is a covering projection if $\wp$ is a local bijection on darts, that is, for each vertex $\tilde{v} \in V_{\tilde{X}}$ the set of darts of $\tilde{X}$ having $\tilde{v}$ as the initial vertex is mapped bijectively onto the set of darts of $X$ having $\wp(\tilde{v})$ as the initial vertex. The preimages $\wp^{-1}(v)\left(v \in V_{X}\right)$ and $\wp^{-1}(x)\left(x \in D_{X}\right)$ are vertex- and dart-fibres, respectively.

Let $\wp_{i}: \tilde{X}_{i} \rightarrow X_{i}(i=1,2)$ be covering projections. A morphism of covering projections $\wp_{1} \rightarrow \wp_{2}$ is an ordered pair of graph morphisms $(\alpha, \tilde{\alpha})$ where $\alpha: X_{1} \rightarrow X_{2}$ and $\tilde{\alpha}: \tilde{X}_{1} \rightarrow$ $\tilde{X}_{2}$ such that $\alpha \wp_{1}=\wp_{2} \tilde{\alpha}$. Note that if $\tilde{\alpha}$ is a covering projection then so is $\alpha$, and if $\alpha$ is a covering projection then so is $\tilde{\alpha}$ provided that $\tilde{\alpha}$ is onto. The definitions of epi-, mono-, isoand automorphisms of covering projections follow directly from the categorical definitions and are self-explanatory. In particular, two covering projections $\wp_{1}$ and $\wp_{2}$ are equivalent if there exists an isomorphism of the form (id, $\phi)$.

The group of covering transformations $\mathrm{CT}(\wp)$ of $\wp: \tilde{X} \rightarrow X$ is the group of all selfequivalences of $\wp$, that is, of all automorphisms $\tau \in$ Aut $\tilde{X}$ such that $\wp \tau=\wp$. Since $\tilde{X}$ is assumed to be connected the group CT( $\wp)$ acts semiregularly on each vertex- and dart-fibre. If CT( $\wp)$ acts transitively (and hence regularly) on each fibre, then the covering projection $\wp$ is called regular. In this case the orbits of CT $(\wp)$ coincide with the fibres of $\wp$, and $\wp$ is isomorphic to the quotient projection $p_{\mathrm{CT}(\wp)}: \tilde{X} \rightarrow \tilde{X}_{\mathrm{CT}(\wp)}$. Moreover, if $X$ is a connected graph and $N \leq$ Aut $X$ acts regularly on each of its vetex- and dart-orbits, then the quotient projection $p_{N}: X \rightarrow X_{N}$ is a regular covering projection with $N$ as the group of covering transformations. An equivalent characterization of a regular covering projection in terms of fundamental groups is the following. Consider the monomorphism $\pi(\tilde{X}, \tilde{b}) \rightarrow \pi(X, b)$ induced by $\wp$, where $\tilde{b} \in \wp^{-1}(b)$. Then $\wp$ is regular if and only if the image of $\pi(\tilde{X}, \tilde{b})$ under this monomorphism is a normal subgroup of $\pi(X, b)$.

\subsection{Voltages}

Regular covering projections can be grasped combinatorially as follows [11, 18]. A Cayley voltage space on a connected graph $X=(D, V$; beg, inv) is an ordered pair $(N ; \zeta)$, where $N$ is a voltage group acting on itself by right multiplication, and $\zeta: D \rightarrow N$ is a function such that $\zeta\left(x^{-1}\right)=(\zeta(x))^{-1}$. The value $\zeta(x)$ is called the voltage of the dart $x \in D$. This function extends naturally to all walks. Note that homotopic walks have the same voltage and that $\zeta: \pi(X, b) \rightarrow N$ is a group homomorphism. Conversely, any homomorphism $\pi(X, b) \rightarrow N$ 'extends' to a voltage space on $X$ by assigning to the darts of a spanning tree the trivial voltage, and to the cotree darts the images of the corresponding generators of $\pi(X, b)$ under this homomorphism. The voltage space is called locally transitive if the homomorphism $\zeta: \pi(X, b) \rightarrow N$ is onto.

Let $(N ; \zeta)$ be a locally transitive Cayley voltage space on a connected graph $X=$ $\left(D, V ;\right.$ beg, inv). With $(N ; \zeta)$ we associate a derived covering projection $\wp_{\zeta}: \operatorname{Cov}(N ; \zeta) \rightarrow$ 
$X$ as follows. The graph $\operatorname{Cov}(N ; \zeta)$ has $D \times N$ and $V \times N$ as the sets of darts and vertices, respectively, with $\operatorname{beg}(x, v)=(\operatorname{beg} x, v)$ and $\operatorname{inv}(x, v)=(\operatorname{inv} x, \nu \zeta(x))$. The corresponding projection $\wp_{\zeta}$ is defined as the projection onto the first component. This is indeed a regular covering projection with $\mathrm{CT}\left(\wp_{\zeta}\right) \cong N$. Namely, for each $a \in N$ define a covering transformation $\lambda_{a}$ by $\lambda_{a}(x, v)=(x, a v)\left(x \in D_{X} \cup V_{X}\right)$. The mapping $\lambda: N \rightarrow \mathrm{CT}\left(\wp_{\zeta}\right)$ establishes an isomorphism of the left regular action of $N$ on itself and the action of CT( $\left.\wp_{\zeta}\right)$ on a fibre. The kernel of the homomorphism $\zeta: \pi(X, b) \rightarrow N$ is precisely the image of $\pi(\tilde{X}, \tilde{b})$ under the monomorphism $\pi(\tilde{X}, \tilde{b}) \rightarrow \pi(X, b)$ induced by $\wp$. Conversely, with any regular covering projection $\wp: \tilde{X} \rightarrow X$ there is an associated locally transitive Cayley voltage space $(N ; \zeta)$ on $X$ with $N \cong \mathrm{CT}(\wp)$ such that the derived covering projection $\wp_{\zeta}$ is equivalent to $\wp$; the required $(N ; \zeta)$ is obtained as follows. First, for each dart $x \in D_{X}$ choose a dart $\tilde{x}_{1} \in \operatorname{fib}(x)$ and label $\tilde{x}_{1}$ and its initial vertex by $\operatorname{lab}\left(\tilde{x}_{1}\right)=\operatorname{lab}\left(\operatorname{beg} \tilde{x}_{1}\right)=1 \in N$. The extension of this labelling to $D_{\tilde{X}}$ and $V_{\tilde{V}}$ obtained by using the left action of CT(§) satisfies the equality $\operatorname{lab}(\tilde{x}) \cdot \operatorname{lab}\left(\operatorname{inv} \tilde{x}_{1}\right)=\operatorname{lab}(\operatorname{inv} \tilde{x})$ for all darts $x \in D_{X}$ and $\tilde{x} \in \operatorname{fib}(x)$. Thus, we set the voltage of the dart $x \in D_{X}$ to be the label of inv $\tilde{x}_{1}$, that is, $\zeta(x)=\operatorname{lab}\left(\operatorname{inv} \tilde{x}_{1}\right.$ ). Moreover, by taking an arbitrary spanning tree $T$ in $X$ and labelling the vertices of a connected component of $\wp^{-1}(T)$ in $\tilde{X}$ by 1 , we obtain a voltage space with the trivial voltage assigned to darts of $T$.

For all group and graph theoretic concepts not defined above we refer the reader to [10, $11,14,18]$.

\section{Lifting and projecting automorphisms}

Let $\wp: \tilde{X} \rightarrow X$ be a covering projection. If $(\alpha, \tilde{\alpha})$ is an automorphism of $\wp$ we say that $\tilde{\alpha}$ is a lift of $\alpha$ and that $\alpha$ is a projection of $\tilde{\alpha}$. Observe that $\alpha$ is uniquely determined by $\tilde{\alpha}$, but $\tilde{\alpha}$ is in general not uniquely determined by $\alpha$. It is true, however, that $\tilde{\alpha}$ is uniquely determined by the image of a single vertex (or dart). The set of all lifts of a given $\alpha \in$ Aut $X$ is denoted by $\mathcal{L}(\alpha)$. Clearly, $\mathcal{L}(\mathrm{id})=\mathrm{CT}(\wp)$. More generally, let $G \leq$ Aut $X$. If for each $\alpha \in G$ the set of lifts $\mathcal{L}(\alpha)$ is nonempty, then $\tilde{G}=\cup_{\alpha \in G} \mathcal{L}(\alpha)$ is a group called the lift of $G$. The induced group homomorphism $\wp_{\tilde{G}}: \tilde{G} \rightarrow G$ has CT(§) as the kernel and hence the sequence $\mathrm{CT}(\wp) \rightarrow \tilde{G} \rightarrow G$ is short exact. We also say that $G$ is a projection of $\tilde{G}$ (although there may exist a proper subgroup of $\tilde{G}$ which projects to $G$ ).

In what follows we restrict to the case when the covering projection $\wp: \tilde{X} \rightarrow X$ is regular. Then the largest subgroup of Aut $\tilde{X}$ that projects is precisely the normalizer of CT(§) within Aut $\tilde{X}$. The question whether a given subgroup $G \leq$ Aut $X$ lifts can be answered in terms of an associated Cayley voltage space $(N, \zeta)$ as follows [18].

Proposition 3.1 Let $(N ; \zeta)$ be a Cayley voltage space associated with a regular covering projection $\wp: \tilde{X} \rightarrow X$. Then a group $G \leq$ Aut $X$ lifts if and only if for each $\alpha \in G$ there exists a group automorphism $\alpha^{\#_{b}} \in$ Aut $N$ such that

$$
\alpha^{\#_{b}} \zeta=\zeta \alpha
$$

where $\zeta$ is considered as a homomorphism defined on $\pi(X, b)$ and $\pi(X, \alpha(b))$, respectively. 
It should be noted that $\alpha^{\#_{b}} \in$ Aut $N$ depends on the base vertex $b \in V_{X}$, and that $\#_{b}: G \rightarrow$ Aut $N$ is not a group homomorphism in general. The situation changes drastically if we assume that the group of covering transformations is abelian. We postpone further discussion on this until Section 5.

Later on we shall need a more general version of Proposition 3.1. This is gathered in the next theorem and its corollary below.

Theorem 3.2 Let $\left(N_{i} ; \zeta_{i}\right)(i=1,2)$ be two Cayley voltage spaces associated with regular covering projections $\wp_{i}: \tilde{X}_{i} \rightarrow X(i=1,2)$, respectively, and let $\alpha \in$ Aut $X$. Then the following statements are equivalent.

(a) There exists an epimorphism $(\alpha, \tilde{\alpha}): \wp_{1} \rightarrow \wp_{2}$.

(b) There exists a group epimorphism $\tau$ : $N_{1} \rightarrow N_{2}$ such that $\tau \zeta_{1}=\zeta_{2} \alpha$, where $\zeta_{1}$ is considered as a homomorphism $\pi(X, b) \rightarrow N_{1}$ and $\zeta_{2}$ as a homomorphism $\pi(X, \alpha(b)) \rightarrow N_{2}$. Moreover, the epimorphism $(\alpha, \tilde{\alpha})$ from the statement $(a)$ is an isomorphism if and only if the epimorphism $\tau$ from statement $(b)$ is an isomorphism.

Proof: We may assume that $\wp_{i}(i=1,2)$ are actually the derived covering projections. Suppose first that (a) holds. Take a closed walk $W$ at $b \in V_{X}$ such that $\zeta_{1}(W)=1$. Its lift $\wp_{1}^{-1}(W)$ is a disjoint union of closed walks isomorphic to $W$. Since $\alpha \wp_{1}=\wp_{2} \tilde{\alpha}$, the image $\tilde{\alpha}\left(\wp_{1}^{-1}(W)\right)$ is a lift of $\alpha(W)$, and hence also a disjoint union of closed walks. Since $\tilde{\alpha}$ is a graph morphism, the cycles in $\tilde{\alpha}\left(\wp_{1}^{-1}(W)\right)$ have length at most that of $W$. On the other hand, being the lifts of $\alpha(W) \cong W$, their length is at least that of $W$. Hence, $\alpha(W)$ lifts to a disjoint union of closed walks isomorphic to $\alpha(W)$, implying that $\zeta_{2}(\alpha(W))=1$. Thus, $\alpha\left(\operatorname{Ker} \zeta_{1}\right) \leq \operatorname{Ker} \zeta_{2}$, and (b) follows.

Suppose now that (b) holds. We first explicitly define the required epimorphism $\tilde{\alpha}$ on darts of $\operatorname{Cov}\left(N_{1}, \zeta_{1}\right)$. Let $(x, v)$ be an arbitrary dart of $\operatorname{Cov}\left(N_{1}, \zeta_{1}\right)$, where $x$ is a dart of $X$ and $v \in N_{1}$. Define

$$
\tilde{\alpha}(x, v)=\left(\alpha(x), \tau(v) \zeta_{2}(\alpha(W))\right),
$$

where $W$ is an arbitrary walk from the base vertex $b$ to beg $x$ with voltage $\zeta_{1}(W)=1$. (Note that such a walk always exists since $\zeta_{1}: \pi(X, b) \rightarrow N_{1}$ is onto.) First of all, the mapping is well defined. Namely, let $W$ and $W^{\prime}$ be two such walks. Then $W^{-1} W^{\prime}$ is a closed walk at $b$ with trivial $\zeta_{1}$-voltage. In view of the fact that $\zeta_{2} \alpha=\tau \zeta_{1}$ the walk $\alpha\left(W^{-1} W^{\prime}\right)$ has trivial $\zeta_{2}$-voltage. Thus, $\zeta_{2}(\alpha(W))=\zeta_{2}\left(\alpha\left(W^{\prime}\right)\right)$, and hence $\tilde{\alpha}$ is well defined. In order to see that it commutes with inv, observe that

$$
\tilde{\alpha}(\operatorname{inv}(x, v))=\left(\alpha\left(x^{-1}\right), \tau(v) \tau\left(\zeta_{1}(x)\right) \zeta_{2}\left(\alpha\left(W^{\prime}\right)\right)\right),
$$

where $W^{\prime}$ is a walk from $b$ to beg $x^{-1}$ with trivial $\zeta_{1}$-voltage. On the other hand,

$$
\operatorname{inv} \tilde{\alpha}(x, v)=\left(\alpha(x)^{-1}, \tau(v) \zeta_{2}(\alpha(W)) \zeta_{2}(\alpha(x))\right)
$$


Let us now consider the closed walk $W^{\prime} x^{-1} W^{-1}$ at $b$. We have $\zeta_{2} \alpha\left(W^{\prime} x^{-1} W^{-1}\right)=$ $\tau \zeta_{1}\left(W^{\prime} x^{-1} W^{-1}\right)$. Using the fact that $\zeta_{1}(W)=\zeta_{1}\left(W^{\prime}\right)=1$, a short calculation now shows that $\tilde{\alpha}$ indeed commutes with inv.

Finally, $\tilde{\alpha}$ can obviously be extended to vertices such that $\tilde{\alpha}$ is indeed a graph epimorphism satisfying $\alpha \wp_{1}=\wp_{2} \tilde{\alpha}$.

The last part of the theorem is clear from the constructions of $\tilde{\alpha}$ and $\tau$.

Corollary 3.3 Let $\left(N_{i} ; \zeta_{i}\right)(i=1,2)$ be two locally transitive Cayley voltage spaces on a connected graph $X$. Then the derived regular covering projections $\wp_{1}$ and $\wp_{2}$ are

(a) isomorphic if and only if there exists a group isomorphism $\tau: N_{1} \rightarrow N_{2}$ and $\alpha \in$ Aut $X$ such that $\tau \zeta_{1}=\zeta_{2} \alpha$, where $\zeta_{1}$ and $\zeta_{2}$ are considered as homomorphisms $\zeta_{1}: \pi(X, b) \rightarrow$ $N_{1}$ and $\zeta_{2}: \pi(X, \alpha(b)) \rightarrow N_{2}$;

(b) equivalent (cf. [23, Theorem 2]) if and only if there exists an isomorphism $\tau: N_{1} \rightarrow N_{2}$ such that $\tau \zeta_{1}=\zeta_{2}$, where $\zeta_{1}$ and $\zeta_{2}$ are considered as homomorphisms defined on $\pi(X, b)$.

\section{Ordering admissible regular coverings}

In this section we revise some results of Venkatesh [24, 25], tailored to our present needs and giving somewhat different proofs.

Let $X$ be a connected graph and $G \leq$ Aut $X$. A regular covering projection $\wp: \tilde{X} \rightarrow X$ is called $G$-admissible if $G$ lifts along $\wp$. If $\wp_{i}: \tilde{X}_{i} \rightarrow X(i=1,2)$ are regular $G$ admissible covering projections, define $\wp_{2} \leq \wp_{1}$ if and only if there exists an epimorphism (id, $\alpha): \wp_{1} \rightarrow \wp_{2}$. Clearly, $\wp_{1}$ and $\wp_{2}$ are equivalent if and only if $\wp_{1} \leq \wp_{2}$ and $\wp_{2} \leq \wp_{1}$. Therefore, the ordering $\leq$ induces a partial ordering on the set $\mathcal{C}_{G}(X)$ of equivalence classes of $G$-admissible regular covering projections onto $X$. This ordering, which is in fact a lattice, is denoted by the same symbol $\leq$. The interval $\left.\left\{\left[\wp^{\prime}\right] \mid\left[\wp^{\prime}\right] \leq[\wp]\right\} \subseteq \mathcal{C}_{G}(X)\right\}$ is denoted by $\mathcal{C}_{G}(\wp)$.

Let $\wp: \tilde{X} \rightarrow X$ be a regular covering projection. If $K \leq \mathrm{CT}(\wp)$, then $\wp$ factors through the regular covering projection $\wp_{K}: \tilde{X} \rightarrow \tilde{X}_{K}$. The corresponding covering projection $\tilde{X}_{K} \rightarrow X$ maps according to the rule $K x \mapsto \wp(x)$, where $K x$ is a dart (or vertex) of $\tilde{X}_{K}$, that is, the orbit of a dart (or vertex) $x$ of $\tilde{X}$ under the action of $K$. If $K$ is normal in $N=\mathrm{CT}(\wp)$ we denote this covering projection by

$$
\wp_{N / K}: \tilde{X}_{K} \rightarrow X .
$$

Since $K$ is normal in $N$, the group $N$ projects along $\wp_{K}$ to a group isomorphic with $N / K$. Because $\wp=\wp_{N / K} \wp_{K}$, this projected group is contained in CT $\left(\wp_{N / K}\right)$ and, moreover, it is transitive on each of the fibres of $\wp_{N / K}$. Thus, $\wp_{N / K}$ is regular with CT $\left(\wp_{N / K}\right)$ isomorphic to $N / K$. If $\wp$ is $G$-admissible with $\tilde{G}$ being the lift of $G$, let $\mathcal{N}_{G}(\wp)$ denote the set of subgroups of $\mathrm{CT}(\wp)$ which are normal in $\tilde{G}$.

Proposition 4.1 Let $(N, \zeta)$ be the Cayley voltage space associated with a $G$-admissible regular covering projection $\wp: \tilde{X} \rightarrow X$, and let $\tilde{G}$ be the lift of $G \leq$ Aut $X$. Suppose that $K \in \mathcal{N}_{G}(\wp)$. Then: 
(a) The covering projection $\wp_{N / K}: \tilde{X}_{K} \rightarrow X$ is regular $G$-admissible, with the lift of $G$ isomorphic to the quotient group $\tilde{G} / K$, and $\wp_{N / K} \leq \wp$;

(b) The covering projection $\wp_{N / K}$ is equivalent to the derived covering projection $\wp_{q \zeta}$ : $\operatorname{Cov}(N / K, q \zeta) \rightarrow X$, where $q: N \rightarrow N / K$ is the natural quotient projection.

Proof: By the remarks above we already know that $\wp_{N / K}$ is regular. Since $K$ is normal in $\tilde{G}$, the group $\tilde{G}$ projects along $\wp_{K}$ onto a group isomorphic with $\tilde{G} / K$. Since $N / K$ is normal in $\tilde{G} / K$, the latter projects along $\wp_{N / K}$. Let $\tilde{g} \in \tilde{G}$ be a lift of $g \in G$ along $\wp$, and let $\bar{g}$ be the projection of $\tilde{g}$ along $\wp_{K}$, that is, let $g \wp=\wp \tilde{g}$ and $\bar{g} \wp_{K}=\wp_{K} \tilde{g}$. Then we have $g \wp_{N / K} \wp_{K}=\wp_{N / K} \wp_{K} \tilde{g}=\wp_{N / K} \bar{g} \wp_{K}$, and consequently, $g \wp_{N / K}=\wp_{N / K} \bar{g}$. Hence $\wp_{N / K}$ is $G$-admissible and $\wp_{N / K} \leq \wp$. This proves (a).

In order to prove (b) we can assume that $\tilde{X}=\operatorname{Cov}(N, \zeta)$ and that $\wp$ is the derived covering projection. The vertices and darts of the graph $\tilde{X}_{K}$ are then the orbits $K(x, v)$, where $x$ is a vertex or a dart of $X$ and $\nu \in N$. Define the mapping $\alpha: \tilde{X}_{K} \rightarrow \operatorname{Cov}(N / K ; q \zeta)$ by $K(x, v) \mapsto(x, K v)$. This is clearly a well defined graph isomorphism satisfying $\wp_{N / K}=$ $\wp_{q \zeta} \alpha$.

The assignment $K \mapsto\left[\wp_{N / K}\right]$, where $K$ and $\wp_{N / K}$ are as in Proposition 4.1, gives rise to a mapping

$$
\Psi_{(G, \wp)}: \mathcal{N}_{G}(\wp) \rightarrow \mathcal{C}_{G}(\wp)
$$

We shall prove that $\Psi_{(G, \wp)}$ is an order reversing bijection (relative to the inclusion-order in $\mathcal{N}_{G}(\wp)$ and the order $\leq$ in $\left.\mathcal{C}_{G}(\wp)\right)$, see Theorem 4.4 below. The bijective correspondence between $\mathcal{N}_{G}(\wp)$ and $\mathcal{C}_{G}(\wp)$ was essentially established in [24, 25], using a somewhat different approach. Theorem 4.4 is a direct consequence of Propositions 4.2 and 4.3 below. The first of these says that the mapping $\Psi_{(G, \wp)}$ is order reversing and mono, while the second one says that $\Psi_{(G, \wp)}$ is onto.

Proposition 4.2 Let $\wp: \tilde{X} \rightarrow X$ be a G-admissible regular covering projection. Let $K_{1}, K_{2} \in \mathcal{N}_{G}(\wp)$ and let $N=\mathrm{CT}(\wp)$. Then $\left[\wp_{N / K_{1}}\right] \leq\left[\wp_{N / K_{2}}\right]$ if and only if $K_{2} \leq K_{1}$. In particular, $\wp_{N / K_{1}}$ and $\wp_{N / K_{2}}$ are equivalent if and only if $K_{1}=K_{2}$.

Proof: Let $(N, \zeta)$ be a Cayley voltage space associated with $\wp$. By Proposition $4.1 \wp_{N / K_{i}}$ $(i=1,2)$ is equivalent to the derived covering projection $\wp_{q_{i} \zeta}: \operatorname{Cov}\left(N / K_{i}, q_{i} \zeta\right) \rightarrow X$, where $q_{i}: N \rightarrow N / K_{i}$ is the natural quotient projection.

Since $\wp_{N / K_{i}}(i=1,2)$ are both $G$-admissible, we have that $\left[\wp_{N / K_{1}}\right] \leq\left[\wp_{N / K_{2}}\right]$ holds if and only if there exists an epimorphism (id, $\alpha$ ): $\wp_{q_{2} \zeta} \rightarrow \wp_{q_{1} \zeta}$. The condition is equivalent, by Theorem 3.2, to the requirement that there is a group epimorphism $\tau: N / K_{2} \rightarrow N / K_{1}$ such that $\tau q_{2} \zeta=q_{1} \zeta$. But this is equivalent to $\tau q_{2}=q_{1}$ (since $\zeta$ is onto), which is nothing but $K_{2}=\operatorname{Ker} q_{2} \leq \operatorname{Ker} q_{1}=K_{1}$. The last statement in the proposition is now evident.

Proposition 4.3 Let $\wp: \tilde{X} \rightarrow X$ and $q: Y \rightarrow X$ be $G$-admissible regular covering projections, where $q \leq \wp$. Then there exists a subgroup $K \in \mathcal{N}_{G}(\wp)$ such that $q$ is equivalent to the covering projection $\wp_{N / K}$, where $N=\mathrm{CT}(\wp)$. 
Proof: By definition there exists a graph epimorphism $\alpha: \tilde{X} \rightarrow Y$ such that $\wp=q \alpha$. Clearly, $\alpha$ is a covering projection. Consider the monomorphic projections of the fundamental groups of $\tilde{X}$ and $Y$ to corresponding subgroups $P$ and $Q$ in $\pi(X, b)$, respectively. Since $\wp$ factors through $q$ we have $P \leq Q$. As $\wp$ is regular, $P$ is normal in $\pi(X, b)$ and hence in $Q$. Thus, the covering projection $\alpha$ is regular, too. It follows that there exists an isomorphism of covering projections $(\iota, \mathrm{id}): \wp_{K} \rightarrow \alpha$, where $K=\mathrm{CT}(\alpha)$. Since $\wp=q \alpha$ we have $K \leq N=\mathrm{CT}(\wp)$. Now let $\bar{G} \leq$ Aut $Y$ be the lift of $G$ along $q$ and let $\tilde{G} \leq$ Aut $\tilde{X}$ be the lift of $G$ along $\wp$. We leave to the reader to show that $\bar{G}$ must lift along $\alpha$ to $\tilde{G}$ (see [?, Lemma 4.1.3]). This is equivalent to saying that $\tilde{G}$ projects along $\wp_{K}$, or, that $K \in \mathcal{N}_{G}(\wp)$. Finally, from $\wp_{N / K} \wp_{K}=\wp=q \alpha=q \iota \wp_{K}$ we conclude that $\wp_{N / K}=q \iota$. In other words, $q$ is equivalent to $\wp_{N / K}: \tilde{X}_{K} \rightarrow X$. (An alternative proof of this proposition using Theorem 3.2 is left to the reader.)

Theorem 4.4 The mapping $\Psi_{(G, \wp)}: \mathcal{N}_{G}(\wp) \rightarrow \mathcal{C}_{G}(\wp)$ is an anti-isomorphism of lattices $\left(\mathcal{N}_{G}(\wp), \subseteq\right)$ and $\left(\mathcal{C}_{G}(\wp), \leq\right)$.

Let $\wp: \tilde{X} \rightarrow X$ be a $G$-admissible regular covering projection. Observe that $\mathcal{C}_{G}(\wp)$ contains at least two equivalence classes, namely the one induced by the identity projection $\operatorname{id}_{X}: X \rightarrow X$ and the one induced by $\wp$. If these are the only elements of $\mathcal{C}_{G}(\wp)$ then $\wp$ is called minimal $G$-admissible regular covering projection [24, 25]. It is clear that any $G$-admissible regular covering can be decomposed into a series of minimal ones. Such coverings can be characterized by a certain maximality condition imposed on the normal subgroups of $\pi(X, b)$ which determine these coverings up to equivalence [24, 25]. An equivalent characterization is the following.

Corollary 4.5 Let $\wp: \tilde{X} \rightarrow X$ be a $G$-admissible regular covering projection. Then $\wp$ is minimal if and only if $\mathrm{CT}(\wp)$ is a minimal normal subgroup of the lifted group $\tilde{G}$.

Proof: By definition, $\wp$ is minimal if and only if the set $\mathcal{C}_{G}(\wp)$ consists of at most two elements. This is equivalent, by Theorem 4 , to saying that the set $\mathcal{N}_{G}(\wp)$ has at most two elements. But this is equivalent to saying that CT(§) is a minimal normal subgroup of $\tilde{G}$.

We end this section by a more general analogue of Proposition 4.2. We give a criterion for the covering projections $\wp_{N / K_{1}}$ and $\wp_{N / K_{2}}$ to be isomorphic. See Sections 6 and 7 for an application.

Proposition 4.6 Let $\wp: \tilde{X} \rightarrow X$ be an (Aut $X$ )-admissible regular covering projection. Suppose that $K_{1}$ and $K_{2}$ are two normal subgroups of $N=\mathrm{CT}(\wp)$. Then the induced covering projections $\wp_{N / K_{1}}: \tilde{X}_{K_{1}} \rightarrow X$ and $\wp_{N / K_{2}}: \tilde{X}_{K_{2}} \rightarrow X$ are isomorphic if and only if there exists an automorphism $\alpha \in$ Aut $X$ such that $\alpha^{\#_{b}}\left(K_{1}\right)=K_{2}$.

Proof: Let $(N, \zeta)$ be a Cayley voltage space associated with $\wp$. By Proposition 4.1, the covering projection $\wp_{N / K_{i}}(i=1,2)$ is associated with the Cayley voltage space $\left(N / K_{i}, q_{i} \zeta\right)$, where $q_{i}: N \rightarrow N / K_{i}$ is the quotient homomorphism. By Corollary 3.3, 
the derived covering projections $\wp_{q_{1} \zeta}$ and $\wp_{q_{2} \zeta}$ are isomorphic if and only if there exists an isomorphism of groups $\tau: N / K_{1} \rightarrow N / K_{2}$ and a graph automorphism $\alpha \in$ Aut $X$ such that $\tau q_{1} \zeta=q_{2} \zeta \alpha$. Since $\alpha$ lifts along $\wp$, Proposition 3.1 implies that there is an automorphism $\alpha^{\#_{b}} \in$ Aut $N$ satisfying $\zeta \alpha=\alpha^{\#_{b}} \zeta$. As $\zeta$ is onto, we deduce that $\tau q_{1} \zeta=q_{2} \zeta \alpha=q_{2} \alpha^{\#_{b}} \zeta$ is equivalent to $q_{2} \alpha^{\#_{b}}=\tau q_{1}$. The existence of $\tau$ is equivalent to the fact that $\alpha^{\#_{b}}$ maps $\operatorname{Ker} q_{1}$ to $\operatorname{Ker} q_{2}$, that is, $\alpha^{\#_{b}}\left(K_{1}\right)=K_{2}$.

\section{Abelian regular covers}

A regular covering projection $\wp: \tilde{X} \rightarrow X$ is abelian if the group CT( $\wp)$ is abelian. Let $(N ; \zeta)$ be a Cayley voltage space associated with such a covering. Observe that the homomorphism $\zeta: \pi(X, b) \rightarrow N$ now factors through the abelianization $\pi(X, b) \rightarrow H_{1}(X)$. The corresponding homomorphism $H_{1}(X) \rightarrow N$ (a linear mapping of $\mathbb{Z}$-modules) will also be denoted by $\zeta$. Converesely, any epimorphism $H_{1}(X) \rightarrow N$ 'extends' to an epimorphism $\pi(X, b) \rightarrow N$ which in turn gives rise to an abelian regular covering projection. We express this informally by saying that the voltage assignment can be taken as defined on the first homology group.

This fact has considerable impact on our treatement of lifting automorphisms. First, we restate Proposition 3.1, omitting the obvious proof. A similar restatement of Theorem 3.2 and of Corollary 3.3 is left to the reader.

Proposition 5.1 Let $(N ; \zeta)$ be a Cayley voltage space associated with an abelian regular covering projection $\wp: \tilde{X} \rightarrow X$. Then a group $G \leq$ Aut $X$ lifts if and only iffor each $\alpha \in G$ there exists a group automorphism $\alpha^{\#} \in$ Aut $N$ such that

$$
\alpha^{\#} \zeta=\zeta \alpha
$$

where $\zeta$ and $\alpha$ are considered as defined on $H_{1}(X)$. In particular, if $G$ lifts then for each $\alpha \in G$ the automorphism $\alpha^{\#_{b}}$ of Proposition 3.1 does not depend on the base vertex $b$, and considered as defined on $H_{1}(X)$ it conicides with $\alpha^{\#}$.

Proposition 5.1 can be further rephrased by saying that the group $G \leq$ Aut $X$ lifts along $\wp_{\zeta}$ if and only if Ker $\zeta$ is an invariant submodule of the naturally defined linear representation of $G$ on the $\mathbb{Z}$-module $H_{1}(X)$. This readily implies that the (equivalence classes of) $G$ admissible abelian regular covering projections of $X$ are in bijective correspondence with the $G$-invariant submodules of finite index in $H_{1}(X)$.

To put the above into a wider setting, recall the anti-isomorphism of lattices $\Psi_{(G, \wp)}$ : $\mathcal{N}_{G}(\wp) \rightarrow \mathcal{C}_{G}(\wp)$ defined in Section 4 . We are now going to show, first, that there is a nice combinatorial characterization of $\mathcal{N}_{G}(\wp)$ in terms of voltages, and second, that this characterization is intimately related with the lifted groups considered as extensions of the group of covering transformations. Suppose that a subgroup $G \leq$ Aut $X$ has a lift. The coupling of the extension $\mathrm{CT}(\wp) \rightarrow \tilde{G} \rightarrow G$ with abelian kernel is a homomorphism $\Theta: G \rightarrow \operatorname{AutCT}(\wp), \alpha \mapsto \Theta_{\alpha}$, defined relative to (and independent of) an algebraic transversal $\{\bar{\alpha} \in \mathcal{L}(\alpha) \mid \alpha \in G\}$ (with id $=\mathrm{id}$ ), where $\Theta_{\alpha}$ is given by $\Theta_{\alpha}: c \mapsto \bar{\alpha} c \bar{\alpha}^{-1}$. 
A general discussion on the coupling of the extension $\mathrm{CT}(\wp) \rightarrow \tilde{G} \rightarrow G$ in terms of fundamental groups can be be found in $[24,25]$.

Theorem 5.2 Let $(N ; \zeta)$ be a locally transitive Cayley voltage space on a connected graph $X$, with $N$ abelian, and let $\wp$ be the derived covering projection. Suppose that $G \leq$ Aut $X$ has a lift. Then:

(a) The induced mapping \#: $G \rightarrow$ Aut $N$ is a group homomorphism.

(b) The coupling for the extension $\mathrm{CT}(\wp) \rightarrow \tilde{G} \rightarrow G$ is $\Theta=\hat{\lambda} \#$, where $\hat{\lambda}$ : Aut $N \rightarrow$ Aut CТ( $\wp)$ is the isomorphism $\phi \mapsto \lambda \phi \lambda^{-1}$ (induced by the isomorphism $\lambda: N \rightarrow$ $\mathrm{CT}(\wp)$ which assigns to each $a \in N$ the covering transformation $\lambda_{a}$ ). In particular, if we identify $N=\mathrm{CT}(\wp)($ via $\lambda)$ then $\alpha^{\#}(\nu)=\tilde{\alpha} \nu \tilde{\alpha}^{-1}$, for each $\alpha \in G, \tilde{\alpha} \in \mathcal{L}(\alpha)$ and $v \in N$.

Proof: Let $\alpha, \beta \in$ Aut $X$, and let $C$ be an arbitrary closed walk in $X$. Then we have $\alpha^{\#} \beta^{\#}(\zeta(C))=\alpha^{\#}(\zeta(\beta(C)))=\zeta(\alpha \beta(C))=(\alpha \beta)^{\#}(\zeta(C))$, and (a) holds.

In order to prove (b), recall that the action of CT(§) on an arbitrary dart or vertex $(x, v)$ of $\operatorname{Cov}(N ; \zeta)$ is given by $\lambda_{a}(x, v)=(x, a v)$, and observe that

$$
\tilde{\alpha}(x, v)=\left(\alpha(x), \tau_{x} \alpha^{\#}(v)\right)
$$

for an appropriate $\tau_{x} \in N$ dependent on $x$ and $\alpha$. We have $\tilde{\alpha} \lambda_{a}(x, v)=\tilde{\alpha}(x, a v)=$ $\left(\alpha(x), \tau_{x} \alpha^{\#}(v)\right)=\left(\alpha(x), \tau_{x} \alpha^{\#}(a) \alpha^{\#}(v)\right)=\left(\alpha(x), \alpha^{\#}(a) \tau_{x} \alpha^{\#}(v)\right)=\lambda_{\alpha^{\#}(a)}\left(\alpha(x), \tau_{x} \alpha^{\#}(v)\right)=$ $\lambda_{\alpha^{\#}(a)} \tilde{\alpha}(x, v)$. Thus, $\tilde{\alpha} \lambda_{a} \tilde{\alpha}^{-1}=\lambda_{\alpha^{\#}(a)}$. It follows that $\left(\Theta_{\alpha} \lambda\right)(a)=\left(\lambda \alpha^{\#}\right)(a)$ for all $a \in N$. Hence $\Theta(\alpha)=\Theta_{\alpha}=\lambda \alpha^{\#} \lambda^{-1}=(\hat{\lambda} \#)(\alpha)$ for all $\alpha \in G$. The rest is obvious.

By part (a) of Theorem 5.2 we can view the mapping \#: $G \rightarrow$ Aut $N$ as a linear representation of the group $G$ over the $\mathbb{Z}$-module $N$. Let $\operatorname{Inv}(\#)$ denote the set of \#-invariant submodules of this representation. Then part (b) of Theorem 5.2 implies the following corollary.

Corollary 5.3 If we identify $N=\mathrm{CT}(\wp)($ via $\lambda)$ as in Theorem 5.2 then $\operatorname{Inv}(\#)=\mathcal{N}_{G}(\wp)$.

In view of the above identification we shall consider the mapping $\Psi_{(G, \wp)}$ as defined on the set $\operatorname{Inv}(\#)$.

As a final remark, consider the homological covering $\hbar: \tilde{X}_{\hbar} \rightarrow X$ which is characterized by the fact that $\mathrm{CT}(\hbar)$ is isomorphic to the first homology group $H_{1}(X)$. Note that the graph $\tilde{X}_{\hbar}$ is infinite and observe that all theorems and propositions stated so far are valid in the infinite case as well. It easily follows that there is, up to equivalence, only one such covering obtained by taking $\zeta: H_{1}(X) \rightarrow H_{1}(X)$ to be the identity mapping. Also, this covering is (Aut $X$ )-admissible. Using Theorem 5.2 we can find all equivalence classes of (finite) $G$ admissible abelian regular covering projections of $X$ via the linear representation of $G$ on $H_{1}(X)$, as already mentioned in the paragraph following Proposition 5.1. We shall take a closer look at this in the next section when discussing $p$-homological covers. 


\section{Elementary abelian regular covers}

We start this section with some remarks regarding notation and terminology. By $\Sigma_{n}$ we denote the standard basis of the (column!) vector space $\mathbb{F}^{n}$ over a field $\mathbb{F}$. If $A: U \rightarrow W$ is a linear mapping between two $\mathbb{F}$-vector spaces $U$ and $W$ of finite dimension, then $\left[A ; \mathcal{B}_{W}, \mathcal{B}_{U}\right]$ denotes the corresponding matrix relative to the bases $\mathcal{B}_{U}$ and $\mathcal{B}_{W}$ for $U$ and $W$, respectively. If $M \in \mathbb{F}^{m \times n}$ is a matrix then the same symbol denotes the corresponding linear mapping $\mathbb{F}^{n} \rightarrow \mathbb{F}^{m}$, that is, $M=\left[M ; \Sigma_{m}, \Sigma_{n}\right]$. By $U^{*}$ we denote the dual of an $\mathbb{F}$-vector space $U$, and by $\mathcal{B}^{*}$ its dual basis relative to a basis $\mathcal{B}$ of $U$. The dual of a linear transformation $A: U \rightarrow U$ is denoted by $A^{*}$. For $K \leq U$ let $\omega(K)=\left\{f \in U^{*} \mid f(K)=0\right\} \leq U^{*}$ denote the annihilator of $K$.

If $\phi: G \rightarrow$ Aut $U$ is a linear representation of a group $G$, then the composition $\phi^{*}=* \phi$ is a linear anti-representation of $G$ on $U^{*}$. By $\operatorname{Inv}(\phi)$ and $\operatorname{Inv}\left(\phi^{*}\right)$ we denote the set of all $\phi$ and $\phi^{*}$-invariant subspaces of these representations, respectively. Observe that $\omega$ induces the anti-isomorphism of lattices $\omega_{\phi}: \operatorname{Inv}(\phi) \rightarrow \operatorname{Inv}\left(\phi^{*}\right)$.

Lemma 6.1 Let $\mathcal{B}=\left\{e_{1}, e_{2}, \ldots, e_{d}\right\}$ be a basis of a vector space $U$ and $\mathcal{B}^{*}=\left\{e_{1}^{*}, e_{2}^{*}, \ldots\right.$, $\left.e_{d}^{*}\right\}$ the dual basis of $U^{*}$. For arbitrary bases $\left\{b_{1}, b_{2}, \ldots, b_{k}\right\}$ and $\left\{f_{1}^{*}, f_{2}^{*}, \ldots, f_{d-k}^{*}\right\}$ of subspaces $K \leq U$ and $M \leq U^{*}$, respectively, let

$$
Q=\left[f_{i}^{*}\left(e_{j}\right)\right]_{i=1, \ldots, d-k}^{j=1, \ldots, d} \in \mathbb{F}^{(d-k) \times d} \quad \text { and } \quad B=\left[e_{i}^{*}\left(b_{j}\right)\right]_{i=1, \ldots, d}^{j=1, \ldots, k} \in \mathbb{F}^{d \times k} .
$$

Then $M=\omega(K)$ if and only if $Q B=0 \in \mathbb{F}^{(d-k) \times k}$.

Proof: It suffices to observe that the $(s, t)^{\text {th }}$-element $[Q B]_{s, t}$ of the matrix $Q B$ is

$$
[Q B]_{s, t}=\sum_{j=1}^{d} f_{s}^{*}\left(e_{j}\right) e_{j}^{*}\left(b_{t}\right)=\left(\sum_{j=1}^{d} f_{s}^{*}\left(e_{j}\right) e_{j}^{*}\right)\left(b_{t}\right)=f_{s}^{*}\left(b_{t}\right) .
$$

From now on we restrict our considerations to finite dimensional vector spaces over an arbitrary prime field $\mathbb{Z}_{p}$, and are ready to consider the main topic of this section: If $X$ is a given connected graph and $G \leq$ Aut $X$ a given subgroup of automorphisms, find all $G$-admissible regular covering projections $\wp: \tilde{X} \rightarrow X$, up to equivalence (or possibly, up to isomorphism) of covering projections. This is almost intractable in general. A restricted subproblem would be to find $\mathcal{C}_{G}\left(\wp_{0}\right)$, where $\wp_{0}$ is some known $G$-admissible covering. In view of Theorem 4.4 we first find the set $\mathcal{N}_{G}\left(\wp_{0}\right)$ (which could be difficult), and then use part (b) of Proposition 4.1 to construct the corresponding coverings $\Psi_{\left(G, \wp_{0}\right)}(K)\left(K \in \mathcal{N}_{G}\left(\wp_{0}\right)\right)$. However, finding the corresponding voltage assignments explicitly - that is, using a suitable predefined presentation of the 'abstract' group $\mathrm{CT}\left(\wp_{0}\right) / K$-may again not be easy. The situation changes if we restrict to consider elementary abelian covers, which we now define.

A regular covering projection $\wp: \tilde{X} \rightarrow X$ is called elementary abelian if the group CT( $\wp)$ is elementary abelian. If $(N ; \zeta)$ is an associated Cayley voltage space we shall sometimes regard the elementary abelian $p$-group $N$ as a $\mathbb{Z}$-module or as the additive group of a vector space over $\mathbb{Z}_{p}$. (Hereafter, $N$ will always denote an elementary abelian $p$-group.) 
As mentioned in Section 5, the commutativity of $N$ enables us to consider the voltage assignment as a linear mapping of $\mathbb{Z}$-modules $\zeta: H_{1}(X) \rightarrow N$. Now even more holds. Since the group $N$ is of exponent $p$, the kernel $\operatorname{Ker} \zeta$ contains the submodule $p H_{1}(X)$, and $\zeta$ factors through the module $H_{1}\left(X ; \mathbb{Z}_{p}\right)$. The induced linear mapping $H_{1}\left(X ; \mathbb{Z}_{p}\right) \rightarrow N$ of vector spaces over $\mathbb{Z}_{p}$ will still be denoted by $\zeta$. Conversely, any such mapping 'extends' to a mapping defined on $\pi(X, b)$, and this defines an appropriate elementary abelian covering. Informally we say that the voltage assignment $\zeta$ can be viewed as defined on the first homology group $H_{1}\left(X ; \mathbb{Z}_{p}\right)$. Also, we leave to the reader to formally adapt all the theorems, propositions and corollaries stated thus far to fit into this context.

Now let $\wp: \tilde{X} \rightarrow X$ be a $G$-admissible elementary abelian regular covering projection, and let $(N ; \zeta)$ be an associated Cayley voltage space with $N \cong \mathbb{Z}_{p}^{d}$, where $\zeta$ is considered as a $\mathbb{Z}_{p}$-linear mapping defined on $H_{1}\left(X ; \mathbb{Z}_{p}\right)$. As usual, \#: $G \rightarrow$ Aut $N$ is the corresponding linear representation and $\Psi_{(G, \wp)}: \operatorname{Inv}(\#) \rightarrow \mathcal{C}_{G}(\wp)$ the anti-isomorphism of lattices. We define $\Psi_{(G, \wp)}^{*}: \operatorname{Inv}\left(\#^{*}\right) \rightarrow \mathcal{C}_{G}(\wp)$ as $\Psi_{(G, \wp)}^{*}=\Psi_{(G, \wp)} \omega_{\#}^{-1}$, where $\omega_{\#}: \operatorname{Inv}(\#) \rightarrow \operatorname{Inv}\left(\#^{*}\right)$ is as above. Observe that $\Psi_{(G, \wp)}^{*}$ is now a lattice isomorphism.

Theorem 6.2 With the notation and assumptions above, let $\varphi: H_{1}\left(X, \mathbb{Z}_{p}\right) \rightarrow \mathbb{Z}_{p}^{r}$ be the isomorphism mapping a chosen basis $\mathcal{C}$ to $\Sigma_{r}$, and let $Z=\left[\zeta ; \Sigma_{d}, \mathcal{C}\right]$.

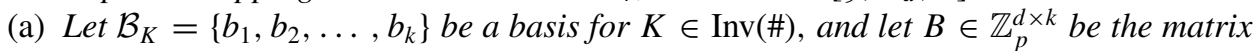
with columns $b_{1}, b_{2}, \ldots, b_{k}$. If $Q \in \mathbb{Z}_{p}^{(d-k) \times d}$ is a matrix of rank $d-k$ satisfying $Q B=0 \in \mathbb{Z}_{p}^{(d-k) \times k}$, then $\wp_{N / K}=\Psi_{(G, \wp)}(K)$ is associated with the Cayley voltage space $\left(\mathbb{Z}_{p}^{d-k} ; Q Z \varphi\right)$.

(b) Let $\Sigma_{d}=\left\{e_{1}, e_{2}, \ldots, e_{d}\right\}$ be the standard basis for $N=\mathbb{Z}_{p}^{d}$, and let $\mathcal{B}_{M}=\left\{f_{1}^{*}, f_{2}^{*}\right.$ $\left., \ldots, f_{m}^{*}\right\}$ be a basis for $M \in \operatorname{Inv}\left(\#^{*}\right)$. Define

$$
Q=\left[f_{i}^{*}\left(e_{j}\right)\right]_{i=1, \ldots, m}^{j=1, \ldots, d} \in \mathbb{Z}_{p}^{m \times d} .
$$

Then $\Psi_{(G, \wp)}^{*}(M)$ is associated with the Cayley voltage space $\left(\mathbb{Z}_{p}^{m} ; Q Z \varphi\right)$.

Proof: We first prove (a). By Proposition 4.1 we can assume that $\tilde{X}_{K}=\operatorname{Cov}(N / K, q \zeta)$ and that $\wp_{N / K}$ is the derived covering projection $\wp_{q \zeta}: \operatorname{Cov}(N / K, q \zeta) \rightarrow X$. Consider the matrices $B$ and $Q$ as linear mappings $\mathbb{Z}_{p}^{k} \stackrel{B}{\longrightarrow} \mathbb{Z}_{p}^{d} \stackrel{Q}{\longrightarrow} \mathbb{Z}_{p}^{d-k}$. Observe that $K=\operatorname{Im} B$. For an arbitrary element $x+K \in N / K$ define $\tau(x+K)=Q x$. Since $Q x=0$ for $x \in K$, the mapping $\tau: N / K \rightarrow \mathbb{Z}_{p}^{d-k}$ is an isomorphism satisfying $\tau q=Q$. Observe that $Z \varphi=\zeta$. Thus, $\tau q \zeta=Q Z \varphi$, and (a) follows by Corollary 3.3.

We now prove (b). Let $\left\{b_{1}, b_{2}, \ldots, b_{d-m}\right\}$ be a basis for $K=\omega^{-1}(M)$ and $\left\{e_{1}^{*}, e_{2}^{*}, \ldots, e_{d}^{*}\right\}$ the dual basis of $\Sigma_{d}$. Define $B=\left[e_{i}^{*}\left(b_{j}\right)\right]_{i=1, \ldots, d}^{j=1, \ldots, d-m} \in \mathbb{Z}_{p}^{d \times(d-m)}$. By Lemma 6.1 we have $Q B=0$, and by part (a) of this theorem, $\Psi_{(G, \wp)}^{*}(M)=\wp_{N / K}$ is associated with $\left(\mathbb{Z}_{p}^{m}, Q Z \varphi\right)$.

Remark In practice, the rows of the matrix $Q$ in (a) of Theorem 6.2 are computed by solving the system of linear equations $B^{t} x=0$.

As for part (b), the $i$ th row of the matrix $Q$ comprises the components of $f_{i}^{*}$ relative to the dual basis $\Sigma_{d}^{*}$. Hence the rows of $Q$ can be computed by finding a basis of an invariant 
subspace of the matrix group $\underline{G}^{t}$, where $\underline{G}$ is a set of matrices which represent the elements of $G^{\#}$ relative to the standard basis $\Sigma_{d}$.

Theorem 6.2 gives rise to an algorithm which explicitly determines all members of the set $\mathcal{C}_{G}(\wp)$ for an arbitrary $G$-admissible elementary abelian regular covering projection $\wp$. If $\wp$ is (Aut $X$ )-admissible, then $\mathcal{C}_{\operatorname{Aut} X}(\wp)$ can be reduced to contain only pairwise noisomorphic coverings.

Proposition 6.3 Let $\wp: \tilde{X} \rightarrow X$ be an (Aut $X$ )-admissible elementary abelian regular covering projection and \#: Aut $X \rightarrow$ Aut CT( $\wp)$ the corresponding linear representation. Further, let $G \leq$ Aut $X$ and let $\#_{G}$ be the restriction of \# to $G$.

(a) Let $K_{1}, K_{2} \in \operatorname{Inv}\left(\#_{G}\right)$. Then the covering projections $\Psi_{(G, p)}\left(K_{1}\right)$ and $\Psi_{(G, p)}\left(K_{2}\right)$ are isomorphic if and only if there exists an automorphism $\alpha \in$ Aut $X$ such that $\alpha^{\#}\left(K_{1}\right)=$ $K_{2}$.

(b) Let $M_{1}, M_{2} \in \operatorname{Inv}\left(\#_{G}^{*}\right)$. Then the covering projections $\Psi_{(G, p)}^{*}\left(M_{1}\right)$ and $\Psi_{(G, p)}^{*}\left(M_{2}\right)$ are isomorphic if and only if there exists an automorphism $\alpha \in \operatorname{Aut} X$ such that $\alpha^{\# *^{*}}\left(M_{1}\right)=$ $M_{2}$.

Proof: Part (a) is a direct consequence of Proposition 4.6. As for part (b), observe that $\alpha^{\# *}(\omega(K))=\omega\left(\alpha^{\#}(K)\right)$, and the result follows.

For a fixed prime $p$ let $\mathcal{C}_{G}^{p}(X) \leq \mathcal{C}_{G}(X)$ denote the set of all equivalence classes of $G$-admissible p-elementary abelian regular covering projections (that is, with the group of covering transformations ranging over all elementary abelian $p$-groups; there are only finitely many such groups corresponding to a given graph $X$ as their dimension is bounded by the requirement that the derived graphs are connected). This set can be explicitly determined from the corresponding homological covering defined below.

A regular covering projection $\tilde{X} \rightarrow X$ is $p$-homological if the group of covering transformations is isomorphic to the first homology group $H_{1}\left(X ; \mathbb{Z}_{p}\right)$. Such a covering projection is generically denoted by $\hbar_{p}: \tilde{X}_{\hbar}^{p} \rightarrow X$ since, by Proposition 6.4 below, it is uniquely determined up to equivalence. Moreover, Proposition 6.4 also shows that its equivalence class is the unique maximal element in the $\operatorname{set} \mathcal{C}_{G}^{p}(X)$.

Proposition 6.4 Let $\hbar_{p}: \tilde{X}_{\hbar}^{p} \rightarrow X$ be a p-homological covering projection. Then $\hbar_{p}$ is (Aut $X$ )-admissible. Moreover, if $\wp: \tilde{X} \rightarrow X$ is a regular covering projection with $\mathrm{CT}(\wp) \cong \mathbb{Z}_{p}^{d}$ (for some $d$ ) then $\wp \leq \hbar_{p}$. In particular, $\hbar_{p}$ is unique, up to equivalence.

Proof: $\quad$ Let $\left(N_{\hbar} ; \zeta_{\hbar}\right)$ be a Cayley voltage space associated with $\hbar_{p}$. As $\zeta_{\hbar}: H_{1}\left(X ; \mathbb{Z}_{p}\right) \rightarrow$ $N_{\hbar}$ is an isomorphism, the group Aut $X$ lifts by Proposition 5.1 (more precisely, by an appropriate version of it).

To prove the second part, let $(N, \zeta)$ be a locally transitive Cayley voltage space associated with $\wp$. Since $\zeta_{\hbar}: H_{1}\left(X, \mathbb{Z}_{p}\right) \rightarrow N_{\hbar}$ is an isomorphism and $\zeta: H_{1}\left(X ; \mathbb{Z}_{p}\right) \rightarrow N$ is onto, there exists an epimorhism $\tau: N_{\hbar} \rightarrow N$ such that $\tau \zeta_{h}=\zeta$. So by Theorem 3.2 (more precisely, by an appropriate version adapted to the context of elementary abelian coverings) there is an epimorphism $\alpha: \tilde{X}_{\hbar}^{p} \rightarrow \tilde{X}$ such that $\wp_{\hbar}=\wp \alpha$, and hence $\wp \leq \wp \hbar$. 
Corollary 6.5 Let $X$ be a connected graph and $G \leq$ Aut $X$. Let $\#_{\hbar_{p}}: G \rightarrow$ Aut $N_{\hbar}$ be the linear representation of $G$ relative to the p-homological covering projection $\hbar_{p}: \tilde{X}_{\hbar}^{p} \rightarrow X$. Then the mapping $\Psi_{\left(G, \hbar_{p}\right)}$ gives rise to a bijective correspondence between the set $\operatorname{Inv}\left(\#_{\hbar_{p}}\right)$ and the set $\mathcal{C}_{G}^{p}(X)$. Moreover, the set $\mathcal{C}_{G}^{p}(X)$ can be found explicitly by the method of Theorem 6.2.

As last remark we note that Theorem 6.2 generalizes Širán̆'s method of finding all cyclic $\mathbb{Z}_{p}$-covers [22]. The problem reduces to finding eigenvectors for $\underline{G}^{t}$, where $\underline{G}$ is the natural linear representation of the group $G \leq$ Aut $X$ on $H_{1}\left(X ; \mathbb{Z}_{p}\right)$.

\section{Examples}

\subsection{Finding minimal invariant subspaces}

Let $X$ be a connected graph, $G$ a subgroup of Aut $X$ and $p$ a prime. The problem of constructing all pairwise non-equivalent $G$-admissible $p$-elementary abelian regular covering projections of $X$ is, by Corollary 6.5, reduced to finding all invariant subspaces of the linear representation $\#_{\hbar_{p}}$ of a group $G$. Clearly, finding such subspaces can be further reduced to finding all common invariant subspaces of the images of generators from some generating set of the group. To the reader's benefit we recall some well known facts related to this topic.

Let $V$ be a vector space of finite dimension over a field $\mathbb{F}$ and let $\phi: G \rightarrow$ Aut $V$ be a linear representation of a group $G$. Let $\alpha \in G$, let $A=\phi(\alpha)$, and let $\kappa_{A}(\lambda)$ and $m_{A}(\lambda)$ be the characteristic and the minimal polynomial of $A$, respectively. Let $\kappa_{A}(\lambda)=$ $f_{1}^{t_{1}}(\lambda) f_{2}^{t_{2}}(\lambda) \ldots f_{k}^{t_{k}}(\lambda)$ and $m_{A}(\lambda)=f_{1}^{s_{1}}(\lambda) f_{2}^{s_{2}}(\lambda) \ldots f_{k}^{s_{k}}(\lambda)$ be the decomposition of the characteristic and the minimal polynomial into irreducible factors, respectively, and let $V_{i}=$ Ker $f_{i}^{s_{i}}(A)$ be the kernel of the transformation $f_{i}^{s_{i}}(A), i \in\{1,2, \ldots, k\}$. The subspaces $V_{i}$, $i \in\{1,2, \ldots, k\}$, are $A$-invariant and $V$ is their direct sum:

$$
V=V_{1} \oplus V_{2} \oplus \cdots \oplus V_{k}
$$

Consider the restrictions $A_{V_{i}}$ of $A$ to $V_{i}(i=1,2, \ldots, k)$. The minimal polynomial of the restriction $A_{V_{i}}$ is $m_{A_{V_{i}}}(\lambda)=f_{i}^{s_{i}}(\lambda)$ (see [14, Chapter IV, Theorem 6]).

Now, let $K$ be an arbitrary $A$-invariant subspace of $V$. The minimal polynomial $m_{A_{K}}(\lambda)$ of the restriction $A_{K}$ of $A$ to $K$ divides $m_{A}(\lambda)$ and is thus of the form $m_{A_{K}}(\lambda)=f_{1}^{r_{1}}(\lambda) f_{2}^{r_{2}}(\lambda)$ $\cdots f_{k}^{r_{k}}(\lambda)$, for some $0 \leq r_{i} \leq s_{i}(i=1,2, \ldots, k)$. By the same argument as above $K$ decomposes as

$$
K=\operatorname{Ker} f_{1}^{r_{1}}\left(A_{K}\right) \oplus \operatorname{Ker} f_{2}^{r_{2}}\left(A_{K}\right) \cdots \oplus \operatorname{Ker} f_{k}^{r_{k}}\left(A_{K}\right)
$$

Since $r_{i} \leq s_{i}(i=1,2, \ldots, k)$ we have $\operatorname{Ker} f_{i}^{r_{i}}\left(A_{K}\right) \leq \operatorname{Ker} f_{i}^{s_{i}}(A) \cap K=V_{i} \cap K$, implying that $K \leq\left(V_{1} \cap K\right) \oplus\left(V_{2} \cap K\right) \oplus \cdots \oplus\left(V_{k} \cap K\right)$. On the other hand, the right-hand side is 
clearly contained in $K$ and so

$$
K=\left(V_{1} \cap K\right) \oplus\left(V_{2} \cap K\right) \oplus \cdots \oplus\left(V_{k} \cap K\right) .
$$

This shows that in order to determine all $A$-invariant subspaces of $V$ it suffices to find all $A$-invariant subspaces of $V_{i}$, for each $i \in\{1,2, \ldots, k\}$, and then take the direct sums of some of them. This can be done by finding the (generalized) Jordan canonical form for each of the linear transformations $A_{V_{i}}$ and the corresponding variety of invariant subspaces. The task can be simplified considerably in the following special case.

Suppose that the characteristic $p$ of the field $\mathbb{F}$ does not divide the order of the transformation $A$. Then by Maschke's Theorem the corresponding $\mathbb{F}\langle A\rangle$-module $V$ is completely reducible, which is by [14, Chapter IV, Theorem 5] equivalent to the fact that the minimal polynomial $m_{A}(\lambda)$ is a product of distinct irreducible factors (with $s_{1}=s_{2}=\ldots=s_{k}=1$ ). If, in adition, $t_{i}=1$ for some $i$, then $V_{i}$ itself is minimal, by [14, Chapter IV, Theorem 3; Chapter III, Theorem 2], and its dimension equals the degree of $f_{i}(\lambda)$. In particular, if $\kappa_{A}(\lambda)=m_{A}(\lambda)$, then all invariant subspaces $V_{i}$ are minimal, and each $A$-invariant subspace of $V$ is a direct sum of some of the subspaces $V_{i}, i \in\{1,2, \ldots, k\}$. These facts will be used in the following concrete example.

\subsection{Elementary abelian covers of prime-dipoles}

Let $q$ be an odd prime and let $X=D_{q}$ be a $q$-dipole on two vertices and $q$ links between them. Then all minimal edge-transitive subgroups of Aut $X$ are cyclic of order $q$, fix the two vertices of $X$ and permute regularly the edges of $X$. Moreover, they are all conjugate in Aut $X$. Let $G$ be such a group and let $\alpha$ be its generator. We shall construct all elementary abelian covers of $X$ such that $G$ lifts. Since each edge-transitive subgroup of Aut $X$ contains a conjugate of $G$, each edge-transitive elementary abelian covering is isomorphic to one obtained in this manner.

Choose a dart $x$ and let $x_{i}=\alpha^{i}(x)$, for each $i \in \mathbb{Z}_{q}$. Let $T$ be the spanning tree of $X$ containig the dart $x_{0}$ and let $b_{i}=x_{i} x_{0}^{-1}(i=1,2, \ldots, q-1)$. See figure 1 .

If $p$ is a prime then $\mathcal{C}=\left\{b_{1}, b_{2}, \ldots, b_{q-1}\right\}$ is a basis of the first homology group $H_{1}=H_{1}\left(X ; \mathbb{Z}_{p}\right)$. Let $\zeta_{\hbar}: H_{1} \rightarrow \mathbb{Z}_{p}^{q-1}$ be the voltage assignment on $X$ mapping $\mathcal{C}$ to the standard basis $\Sigma_{q-1}$, let $\hbar: \tilde{X}_{\hbar} \rightarrow X$ be the derived $p$-homological covering projection, and let \#: $G \rightarrow$ Aut $\mathbb{Z}_{p}^{q-1}$ be the corresponding linear representation of $G$.

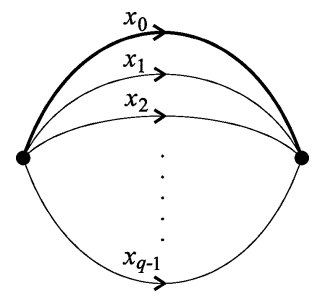

Figure 1. The $q$-dipole $D_{q}$. 
In order to find all $G$-admissible $p$-elementary abelian regular covering projections we have to find, by Theorem 6.2(b), all invariant subspaces of the transpose of the matrix

$$
R=\left[\alpha^{\#} ; \Sigma_{q-1}, \Sigma_{q-1}\right]=\left(\begin{array}{lllll}
-1 & -1 & -1 & \cdots & -1 \\
1 & 0 & 0 & \cdots & 0 \\
0 & 1 & 0 & \cdots & 0 \\
\vdots & \ddots & \ddots & \ddots & \vdots \\
0 & \cdots & 0 & 1 & 0
\end{array}\right)
$$

Observe that $m_{R^{t}}(\lambda)=\kappa_{R^{t}}(\lambda)=\lambda^{q-1}+\lambda^{q-2}+\cdots+1$. By the remarks at the beginning of this section we need to decompose the above polynomial into irreducible factors in the polynomial ring $\mathbb{Z}_{p}[\lambda]$. There are two cases to be considered according to whether $p \neq q$ or $p=q$.

Suppose that $p \neq q$. Let $E^{(q)}$ denote the set of primitive $q$-roots of unity in the splitting field of $\lambda^{q}-1$ over $\mathbb{Z}_{p}$. The multiplicative group $\mathbb{Z}_{q}^{*}$ acts on $E^{(q)}$ in the natural way according to the rule $\xi \mapsto \xi^{r}$, for $r \in \mathbb{Z}_{q}^{*}$ and $\xi \in E^{(q)}$. Let $\bar{p} \in \mathbb{Z}_{q}^{*}$ be the residue class of the prime $p$ modulo $q$, and let $o$ be the order of the group $P=\langle\bar{p}\rangle \leq \mathbb{Z}_{q}^{*}$. Furthermore, let $\xi_{1}, \xi_{2}, \ldots \xi_{m}$, where $m=(q-1) / o$, be the representatives of the orbits of the action of $P$ on $E^{(q)}$. For $i \in\{1,2, \ldots, m\}$, let

$$
f_{i}(\lambda)=\left(\lambda-\xi_{i}\right)\left(\lambda-\xi_{i}^{\bar{p}}\right)\left(\lambda-\xi_{i}^{\bar{p}^{2}}\right) \cdots\left(\lambda-\xi_{i}^{\bar{p}^{o-1}}\right) .
$$

Note that the coefficients of $f_{i}(\lambda)$ belong to $\mathbb{Z}_{p}$ and that

$$
\lambda^{q-1}+\lambda^{q-2}+\cdots+1=f_{1}(\lambda) f_{2}(\lambda) \cdots f_{m}(\lambda)
$$

is the decomposition of $m_{R^{t}}(\lambda)$ into irreducible factors in $\mathbb{Z}_{p}[\lambda]$; in particular, $m_{R^{t}}(\lambda)$ is irreducible if and only if $\bar{p}$ is a generator of $\mathbb{Z}_{q}^{*}$ (see [15, Theorem 2.47]).

Since the minimal and the characteristic polynomial of $R^{t}$ coincide, all invariant subspaces of $R^{t}$ are obtained as direct sums of the minimal invariant subspaces $M_{i}=\operatorname{Ker} f_{i}\left(R^{t}\right)$ $(i=1,2, \ldots, m)$, each being of dimension $o$. The above shows that there are exactly $m$ non-equivalent minimal $G$-admissible $p$-elementary abelian regular covering projections over $X$, with the group of covering transformations isomorphic to $\mathbb{Z}_{p}^{o}$. We shall now prove, using Proposition 6.2, that they are pairwise isomorphic.

To this end, pick $i \in\{1,2, \ldots, m\}$ arbitrarily, and let $k \in \mathbb{Z}_{q}^{*}$ be such that $\xi_{i}^{k}=\xi_{1}$. The mapping of the darts of $X$ defined by $\beta_{k}\left(x_{i}\right)=x_{k i}$ extends to an automorphism $\beta_{k} \in$ Aut $X$. It is easy to check that $\beta \alpha \beta^{-1}=\alpha^{k}$, and so the matrix $S_{k}=\left[\beta^{\#} ; \Sigma_{q-1}, \Sigma_{q-1}\right]$ satisfies $S_{k}^{-t} R^{t} S_{k}^{t}=\left(R^{t}\right)^{k}$. Therefore,

$$
S_{k}^{-t} M_{1}=S_{k}^{-t} \operatorname{Ker} f_{1}\left(R^{t}\right)=\operatorname{Ker} f_{1}\left(\left(R^{t}\right)^{k}\right) .
$$


Note that the above computation holds for an arbitrary $k \in \mathbb{Z}_{q}^{*}$. However, by our particular choice of $k$ we have

$$
\begin{aligned}
f_{1}\left(\lambda^{k}\right) & =\left(\lambda^{k}-\xi_{1}\right)\left(\lambda^{k}-\xi_{1}^{\bar{p}}\right)\left(\lambda^{k}-\xi_{1}^{\bar{p}^{2}}\right) \ldots\left(\lambda^{k}-\xi_{1}^{\bar{p}^{o-1}}\right) \\
& =\left(\lambda^{k}-\xi_{i}^{k}\right)\left(\lambda^{k}-\xi_{i}^{\bar{p} k}\right)\left(\lambda^{k}-\xi_{i}^{\bar{p}^{2} k}\right) \ldots\left(\lambda^{k}-\xi_{i}^{\bar{p}^{o-1} k}\right) \\
& =\left(\lambda-\xi_{i}\right) g_{0}(\lambda)\left(\lambda-\xi_{i}^{\bar{p}}\right) g_{1}(\lambda) \ldots\left(\lambda-\xi_{i}^{\bar{p}^{o-1}}\right) g_{(o-1)}(\lambda) \\
& =f_{i}(\lambda) g(\lambda)
\end{aligned}
$$

for some polynomial $g(\lambda) \in \mathbb{Z}_{p}[\lambda]$. This implies

$$
M_{i}=\operatorname{Ker} f_{i}\left(R^{t}\right) \subseteq \operatorname{Ker} f_{1}\left(\left(R^{t}\right)^{k}\right)=S_{k}^{-t} M_{1}
$$

Moreover, since $S_{k}$ in invertible the above inclusion is in fact equality, and by Proposition 6.3 the covering projections $\Psi_{\left(G, \wp_{\hbar}\right)}^{*}\left(M_{i}\right)$ and $\Psi_{\left(G, \wp_{\hbar}\right)}^{*}\left(M_{1}\right)$ are isomorphic.

Finally, suppose that $p=q$. Now, the minimal polynomial decomposes as $m_{R^{t}}(\lambda)=$ $(\lambda-1)^{q-1}$, and so the Jordan canonical form of $R^{t}$ is just a single elementary Jordan matrix. It is easy to see that the nontrivial invariant subspaces of $R^{t}$ are then

$$
\operatorname{Ker}\left(R^{t}-I\right) \subseteq \operatorname{Ker}\left(R^{t}-I\right)^{2} \subseteq \cdots \subseteq \operatorname{Ker}\left(R^{t}-I\right)^{q-1}=\mathbb{Z}_{p}^{q-1} .
$$

In particular, $\operatorname{Ker}\left(R^{t}-I\right)$ is 1 -dimensional with $(1,2, \ldots, q-1)^{t}$ as its base vector. Hence the covering projection corresponding to $\operatorname{Ker}\left(R^{t}-I\right)$ is associated with the voltage assignment $\zeta\left(x_{i}\right)=i(i=0,1, \ldots, q-1)$ valued in $\mathbb{Z}_{p}$. The derived covering graph is isomorphic to the complete bipartite graph $K_{p, p}$.

Let $\tau \in$ Aut $D_{q}$ be the automorphism switching the two vertices and fixing all edges. Then any subspace of $\mathbb{Z}_{p}^{q-1}$ is invariant under the action of $-I=\left[\tau^{\#} ; \Sigma_{q-1}, \Sigma_{q-1}\right]$. So regardless of whether $p \neq q$ or $p=q$, the automorphism $\tau$ lifts along any $G$-admissible $p$-elementary abelian regular covering projection. Thus, the corresponding covering graphs are arc-transitive.

To close the example we consider two interesting special cases, namely, $q=3$ and $q=7$, $p=2$.

Special case $\mathbf{q}=3$. If $p=3$ then $m_{R^{t}}(\lambda)=(\lambda-1)^{2}$, and the only nontrivial and nonhomological $G$-admissible $p$-elementary abelian regular covering projection of $X$ is, up to equivalence, the cyclic $\mathbb{Z}_{3}$-cover defined by $\zeta\left(x_{0}\right)=0, \zeta\left(x_{1}\right)=1$ and $\zeta\left(x_{2}\right)=2$. The corresponding covering graph is isomorphic to $K_{3,3}$.

If $p \equiv-1(\bmod 3)$ then the minimal polynomial $m_{R^{t}}(\lambda)=\lambda^{2}+\lambda+1$ is irreducible, and the only nontrivial $p$-elementary abelian $G$-admissible cover of $X$ is the homological one.

If $p \equiv 1(\bmod 3)$ then there is a nontrivial element $\xi \in \mathbb{Z}_{p}$ satisfying $\xi^{3}=1$, and the minimal polynomial decomposes as $m_{R^{t}}(\lambda)=(\lambda-\xi)\left(\lambda-\xi^{2}\right)$. The kernels of $R^{t}-\xi I$ and $R^{t}-\xi^{2} I$ are, respectively,

$$
\operatorname{Ker}\left(R^{t}-\xi I\right)=\left\langle\left(1,-\xi^{2}\right)^{t}\right\rangle \quad \text { and } \operatorname{Ker}\left(R^{t}-\xi^{2} I\right)=\left\langle(1,-\xi)^{t}\right\rangle
$$

So apart from the trivial and the homological covering projection there are two additional non-equivalent $G$-admissible $p$-elementary abelian coverings, namely, the cyclic $\mathbb{Z}_{p}$-covers 



Figure 2. The minimal covers of $D_{3}$ in case $p \equiv 1(\bmod 3)$.

derived from the voltage assignments shown in figure 2:

$$
\begin{aligned}
& \zeta_{1}\left(x_{0}\right)=0, \quad \zeta_{1}\left(x_{1}\right)=1, \quad \zeta_{1}\left(x_{2}\right)=-\xi^{2}, \\
& \zeta_{2}\left(x_{0}\right)=0, \quad \zeta_{2}\left(x_{1}\right)=1, \quad \zeta_{2}\left(x_{2}\right)=-\xi .
\end{aligned}
$$

As already discussed above these two covering projections are isomorphic; in particular, the derived graphs are isomorphic.

We remark that along the above two covering projections no subgroup of Aut $D_{3}$ acting 2-transitively on the set of edges lifts. For otherwise, an automorphism fixing an edge and swapping the other two would lift. To see that this is not possible is left to the reader.

Special case $\mathbf{q}=\mathbf{7}, \mathbf{p}=\mathbf{2}$. In this case the minimal polynomial $m_{R^{t}}(\lambda)$ decomposes as

$$
m_{R^{t}}(\lambda)=\left(\lambda^{3}+\lambda+1\right)\left(\lambda^{3}+\lambda^{2}+1\right) .
$$

So apart from the trivial and the homological covering projection, there are also two $G$ admissible $p$-elementary abelian regular covering projections, with the group of covering transformations isomorphic to $\mathbb{Z}_{2}^{3}$, obtained from the kernels

$$
\begin{aligned}
\operatorname{Ker}\left(\left(R^{t}\right)^{3}+R^{t}+I\right)=\langle & (1,0,0,0,1,1)^{t}, \\
& (0,1,0,1,1,1)^{t}, \\
& \left.(0,0,1,1,0,1)^{t}\right\rangle \text { and } \\
\operatorname{Ker}\left(\left(R^{t}\right)^{3}+\left(R^{t}\right)^{2}+I\right)=\left\langle(1,0,0,1,1,1)^{t},\right. & (0,1,0,1,1,0)^{t}, \\
& \left.(0,0,1,0,1,1)^{t}\right\rangle .
\end{aligned}
$$

The respective non-equivalent but, however, isomorphic covering projections are associated with the Cayley voltage space defined by the voltage assignments below.

\begin{tabular}{llllllll}
\hline & $x_{0}$ & $x_{1}$ & $x_{2}$ & $x_{3}$ & $x_{4}$ & $x_{5}$ & $x_{6}$ \\
$\zeta_{1}$ & $\left(\begin{array}{l}0 \\
0 \\
0\end{array}\right)$ & $\left(\begin{array}{l}1 \\
0 \\
0\end{array}\right)$ & $\left(\begin{array}{l}0 \\
1 \\
0\end{array}\right)$ & $\left(\begin{array}{l}0 \\
0 \\
1\end{array}\right)$ & $\left(\begin{array}{l}0 \\
1 \\
1\end{array}\right)$ & $\left(\begin{array}{l}1 \\
1 \\
0\end{array}\right)$ & $\left(\begin{array}{l}1 \\
1 \\
1\end{array}\right)$ \\
$\zeta_{2}$ & $\left(\begin{array}{l}0 \\
0 \\
0\end{array}\right)$ & $\left(\begin{array}{l}1 \\
0 \\
0\end{array}\right)$ & $\left(\begin{array}{l}0 \\
1 \\
0\end{array}\right)$ & $\left(\begin{array}{l}0 \\
0 \\
1\end{array}\right)$ & $\left(\begin{array}{l}1 \\
1 \\
0\end{array}\right)$ & $\left(\begin{array}{l}1 \\
1 \\
1\end{array}\right)$ & $\left(\begin{array}{l}1 \\
0 \\
1\end{array}\right)$ \\
\hline
\end{tabular}



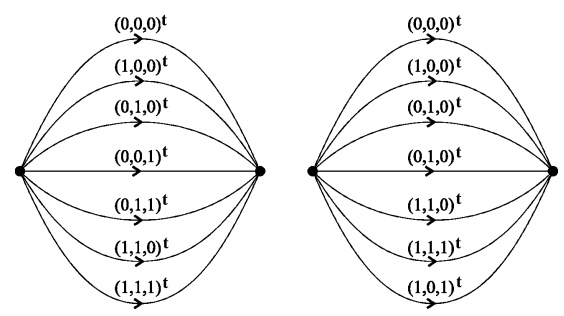

Figure 3. The minimal covers of $D_{7}$ for $p=2$.

The intriguing point of this example is the fact that all 2-elementary abelian minimal $G$-admissible covers of $D_{7}$ are in fact 2 -arc transitive. To this end, observe that the above minimal invariant subspaces of $R^{t}$ are invariant for $\left[\gamma^{\#} ; \Sigma_{6}, \Sigma_{6}\right]^{t}$, where $\gamma=$ $\left(x_{1} x_{2}\right)\left(x_{3} x_{6}\right)\left(x_{1}^{-1} x_{2}^{-1}\right)\left(x_{3}^{-1} x_{6}^{-1}\right) \in$ Aut $D_{7}$. Thus $\gamma$ has a lift. Now the group $\langle\alpha, \gamma\rangle$ is isomorphic to PSL $(3,2)$ and acts 2 -transitively on the set of edges of $D_{7}$, and the claim follows.

\subsection{Semisymmetric elementary abelian covers of the Heawood graph}

Edge-transitive graphs and more specifically, semisymmetric graphs (regular edge- but not vertex-transitive graphs), have recently received a wide attention. Regular covers, and elementary abelian in particular, have proved to be very useful in this context. Let us call a covering projection $\wp: \tilde{X} \rightarrow X$ vertex-transitive (edge-transitive), if a vertex-transitive (edge-transitive) subgroup of Aut $X$ lifts along $\wp$. If $\wp$ is edge- but not vertex-transitive, then $\wp$ is called semisymmetric. Observe that the derived graph of a semisymmetric covering projection is a good candidate for a semisymmetric graph. Namely, the only additional condition such a graph has to satisfy is that the fibres are blocks of imprimitivity of its full automorphism group.

In this subsection we compute, up to isomorphism of covering projections, all those (connected) semisymmetric $p$-elementary abelian regular covering projections $\wp: \tilde{X} \rightarrow \mathcal{H}$, where $\mathcal{H}$ is the Heawood graph. (We acknowledge the use of Magma [?].) Recall that the vertex set of the Heawood graph $\mathcal{H}$ can be identified with the set $\mathbb{Z}_{7} \times \mathbb{Z}_{2}$ in such a way that, for each $i \in \mathbb{Z}_{7}$, the vertex $(i, 0) \in V(\mathcal{H})$ is adjacent to vertices $(i+1,1),(i+2,1)$, and $(i+4,1)$. The automorphism group Aut $\mathcal{H}$ is isomorphic to the group PGL(2, 7) (of order 336), and can be generated by the following permutations:

$$
\begin{aligned}
\rho & :(i, j) \mapsto(i+1, j), \quad i \in \mathbb{Z}_{7}, j \in \mathbb{Z}_{2}, \\
\sigma & :(i, j) \mapsto(2 i, j), \quad i \in \mathbb{Z}_{7}, j \in \mathbb{Z}_{2}, \\
\tau: & (i, j) \mapsto(-i, j+1), \quad i \in \mathbb{Z}_{7}, j \in \mathbb{Z}_{2}, \\
\omega & =((1,0),(5,0))((4,0),(6,0))((0,1),(5,1))((3,1),(6,1)) .
\end{aligned}
$$

Moreover, Aut $\mathcal{H}$ contains precisely one conjugacy class of minimal edge-transitive subgroups (which are also semisymmetric), with the group $H=\langle\rho, \sigma\rangle$ of order 21 as its representative. The group $H$ is maximal in the maximal semisymmetric subgroup of Aut $\mathcal{H}$ 
(isomorphic to PSL $(2,7)$ ). Further, there is exactly one minimal arc-transitive subgroup of Aut $\mathcal{H}$ containing $H$, namely its normalizer $G=N_{\text {Aut } \mathcal{H}}(H)=\langle H, \tau\rangle$ in Aut $\mathcal{H}$. The order of $G$ is 42 and moreover, $G$ is a maximal subgroup of Aut $\mathcal{H}$. In order to find, up to isomorphism, all (connected) edge-transitive elementary abelian regular covering projections of the Heawood graph it suffices to compute all $H$-admissible elementary abelian coverings-for a covering projection is edge-transitive if and only if some minimal edge-transitive group lifts, $H$ is unique up to conjugation, and covering projections along which two conjugate groups lift are isomorphic. Moreover, by identifying those $H$-admissible covering projections which are not $\tau$-admissible, all semisymmetric elementary abelian regular covering projections of the Heawood graph are obtained. Namely, if some vertex-transitive subgroup lifts, then there is an arc-transitive group containing $H$ which lifts. So $G$ would have to lift, a contradiction. That it is enough to require that $\tau$ does not lift follows from the fact that $H$ is of index 2 in $G$. We may now proceed with the computations. Let $\mathcal{T}$ be the spanning tree of $\mathcal{H}$ induced by the edges

$$
\{(i, 0),(i+1,1)\}, i \in \mathbb{Z}_{7} \backslash\{5\}, \quad \text { and } \quad\{(i, 0),(i+2,1)\}, i \in \mathbb{Z}_{7}
$$

This tree is shown in Figure 4, with a simplified vertex notation $i=(i, 0)$ and $i^{\prime}=(i, 1)$. Further, let the dart $(5,0) \rightarrow(6,1)$ be denoted by $a_{7}$, and for each $i \in \mathbb{Z}_{7}$, let $a_{i}$ denote the dart $(i, 0) \rightarrow(i+4,1)$. For simplicity reasons we let the symbol $a_{j}, j \in\{0,1, \ldots, 7\}$, denote also the element of the homology group $H_{1}\left(\mathcal{H} ; \mathbb{Z}_{p}\right)$ induced by the dart $a_{j}$ relative to $\mathcal{T}$. Let $\mathcal{B}$ be the (ordered) basis of $H_{1}\left(\mathcal{H} ; \mathbb{Z}_{p}\right)$ containing the elements $a_{7}, a_{0}, a_{1}, \ldots, a_{6}$ (in this order). Let $\zeta_{\hbar}: H_{1}\left(\mathcal{H} ; \mathbb{Z}_{p}\right) \rightarrow \mathbb{Z}_{p}^{8}$ be the voltage assignment on $X$ mapping $\mathcal{B}$ to the standard basis $\Sigma_{8}$ of $\mathbb{Z}_{p}^{8}$, and let $\hbar: \tilde{\mathcal{H}}_{\hbar} \rightarrow \mathcal{H}$ be the derived $p$-homological covering projection. Further, let $\#$ : Aut $\mathcal{H} \rightarrow$ Aut $\mathbb{Z}_{p}^{8}$ be the corresponding linear representation of Aut $\mathcal{H}$.

By Corollary 6.5, in order to find all $H$-admissible $p$-elementary abelian regular covering projections, we have to find all common invariant subspaces of matrices $R=\left(\rho^{\#}\right)^{t}=\rho^{\# *}$
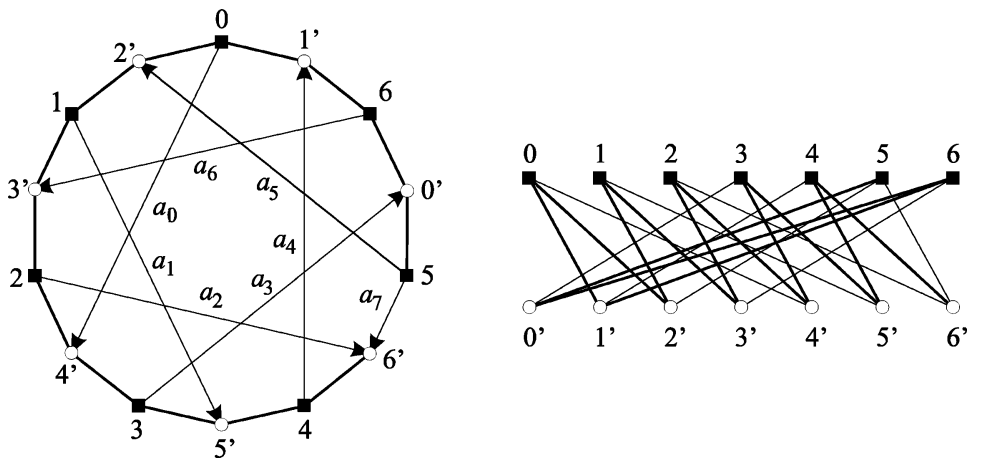

Figure 4. Two drawings of the Heawood graph with a chosen spanning tree. 
and $S=\left(\sigma^{\#}\right)^{t}=\sigma^{\#^{*}}$. A direct computation shows that

$$
R=\left(\begin{array}{cccccccc}
1 & 0 & 0 & 0 & 0 & 0 & 0 & 0 \\
0 & 0 & 1 & 0 & 0 & 0 & 0 & 0 \\
0 & 0 & 0 & 1 & 0 & 0 & 0 & 0 \\
1 & 0 & 0 & 0 & 1 & 0 & 0 & 0 \\
0 & 0 & 0 & 0 & 0 & 1 & 0 & 0 \\
-1 & 0 & 0 & 0 & 0 & 0 & 1 & 0 \\
0 & 0 & 0 & 0 & 0 & 0 & 0 & 1 \\
0 & 1 & 0 & 0 & 0 & 0 & 0 & 0
\end{array}\right) \quad S=\left(\begin{array}{cccccccc}
0 & -1 & -1 & -1 & -1 & -1 & -1 & -1 \\
0 & -1 & 0 & -1 & 0 & -1 & 0 & 0 \\
0 & 0 & 0 & -1 & 0 & -1 & 0 & -1 \\
0 & 0 & -1 & 0 & 0 & -1 & 0 & -1 \\
0 & 1 & 0 & 1 & 0 & 1 & 1 & 0 \\
0 & 1 & 0 & 1 & 0 & 1 & 0 & 1 \\
0 & -1 & 0 & 0 & -1 & 0 & -1 & 0 \\
1 & -1 & 0 & -1 & 0 & 0 & -1 & 0
\end{array}\right)
$$

We start by determining the (minimal) $R$-invariant subspaces. First, the characteristic and the minimal polynomial of the matrix $R$ over the field $\mathbb{Z}_{p}$ are $\kappa(x)=(x-1)\left(x^{7}-1\right)$ and $m(x)=\left(x^{7}-1\right)$, respectively. Let $\mathbb{F}$ denote the splitting field of $m(x)$ over $\mathbb{Z}_{p}$, and let $\xi$ be a primitive 7 th root of unity in $\mathbb{Z}_{p}$. In $\mathbb{F}$, the polynomial $m(x)$ factorizes as

$$
m(x)=\prod_{k=0}^{6}\left(x-\xi^{k}\right),
$$

while the factorization of $m(x)$ over $\mathbb{Z}_{p}$ depends on the congruence class of $p$ modulo 7 . To express these factorizations explicitly, let us define $\alpha_{1}=\xi+\xi^{2}+\xi^{4}, \alpha_{2}=\xi^{3}+\xi^{5}+\xi^{6}$, $\beta_{1}=\xi^{1}+\xi^{6}, \beta_{2}=\xi^{2}+\xi^{5}$ and $\beta_{3}=\xi^{3}+\xi^{4}$ By [?, Theorems 2.47 and 2.14] we have the following cases:

(i) $p \equiv 1(\bmod 7)$. Then $\xi \in \mathbb{Z}_{p}$ and $m(x)$ factorizes into linear factors

$$
m(x)=\prod_{k=0}^{6}\left(x-\xi^{k}\right) \text {. }
$$

(ii) $p \equiv 2$ or $4(\bmod 7)$. Then $\xi \notin \mathbb{Z}_{p}$ but $\alpha_{1} \in \mathbb{Z}_{p}$ and $\alpha_{2} \in \mathbb{Z}_{p}$, and $m(x)$ decomposes as $m(x)=(x-1)\left((x-\xi)\left(x-\xi^{2}\right)\left(x-\xi^{4}\right)\right)\left(\left(x-\xi^{3}\right)\left(x-\xi^{5}\right)\left(x-\xi^{6}\right)\right)$, or

$$
m(x)=(x-1)\left(x^{3}-\alpha_{1} x^{2}+\alpha_{2} x-1\right)\left(x^{3}-\alpha_{2} x^{2}+\alpha_{1} x-1\right) .
$$

(iii) $p \equiv 3$ or $5(\bmod 7)$. Then the polynomial $x^{6}+x^{5}+x^{4}+x^{3}+x^{2}+x+1$ is irreducible and so $m(x)$ decomposes as

$$
m(x)=(x-1)\left(x^{6}+x^{5}+x^{4}+x^{3}+x^{2}+x+1\right) .
$$

(iv) $p \equiv 6(\bmod 7)$. Then $\xi \notin \mathbb{Z}_{p}$ while $\beta_{i} \in \mathbb{Z}_{p}$, for $i \in\{1,2,3\}$, and the polynomial $m(x)$ decomposes as $m(x)=(x-1)\left((x-\xi)\left(x-\xi^{6}\right)\right)\left(\left(x-\xi^{2}\right)\left(x-\xi^{5}\right)\right)\left(\left(x-\xi^{3}\right)\left(x-\xi^{4}\right)\right)$, or

$$
m(x)=(x-1)\left(x^{2}-\beta_{1} x+1\right)\left(x^{2}-\beta_{2} x+1\right)\left(x^{2}-\beta_{3} x+1\right) .
$$

(v) $p=7$. Then the polynomial $m(x)$ decomposes as

$$
m(x)=(x-1)^{7}
$$


Detailed computations are given solely for the case $p \equiv 1(\bmod 7)$. The other cases can be carried out in a similar fashion. In view of Section 7.1 and by direct computation it follows that the minimal $R$-invariant subspaces are:

$$
K_{i}=\operatorname{Ker}\left(R-\xi^{i} I\right)=\left\langle\left(0,1, \xi^{i}, \xi^{2 i}, \xi^{3 i}, \xi^{4 i}, \xi^{5 i}, \xi^{6 i}\right)^{t}\right\rangle, \quad i \in\{1,2, \ldots, 6\}
$$

and the 1-dimensional subspaces of

$$
K_{0}=\operatorname{Ker}(R-I)=\left\langle(1,0,0,0,-1,-1,0,0)^{t},(0,1,1,1,1,1,1,1)^{t}\right\rangle .
$$

In order to find the minimal $\langle R, S\rangle$-invariant subspaces we now consider the action of $S$ on the above $R$-invariant subspaces. First observe that $S$ acts on the $\left\{K_{i} \mid i \in\right.$ $\{0,1, \ldots, 6\}$ as the permutation $S:\left(K_{0}\right)\left(K_{1}, K_{2}, K_{4}\right)\left(K_{3}, K_{6}, K_{5}\right)$. This immediately implies the following: There are exactly two minimal $\langle R, S\rangle$-invariant subspaces contained in $K_{1}+K_{2}+\cdots+K_{6}$, namely

$$
W_{1}=K_{1}+K_{2}+K_{4}, \quad \text { and } \quad W_{2}=K_{3}+K_{5}+K_{6}
$$

As for the subspace $K_{0}$, which is invariant for $S$ as well, it can be minimal or not, depending on the congruence class of the prime $p$ modulo 3 . Indeed, $K_{0}$ is a minimal $S$-invariant subspace in case $p \equiv 2(\bmod 3)$, and there are exactly two $S$-invariant 1 -dimensional subspaces of $K_{0}$ in case $p \equiv 1(\bmod 3)$. If $\lambda$ is a root of the polynomial $x^{2}+x+1 \in \mathbb{Z}_{p}[x]$, then these subspaces are

$$
\begin{aligned}
& L_{1}=\left\langle(\lambda+3,1,1,1,-\lambda-2,-\lambda-2,1,1)^{t}\right\rangle, \\
& L_{2}=\left\langle\left(\lambda^{2}+3,1,1,1,-\lambda^{2}-2,-\lambda^{2}-2,1,1\right)^{t}\right\rangle .
\end{aligned}
$$

We conclude that the minimal $\langle R, S\rangle$-invariant subspaces are $K_{0}, W_{1}$ and $W_{2}$ in case $p \equiv$ $2(\bmod 3)$, and $L_{1}, L_{2}, W_{1}$ and $W_{2}$ in case $p \equiv 1(\bmod 3)$. Other invariant subspaces are obtained by taking direct sums of the minimal ones, that is, $K_{0}+W_{1}, K_{0}+W_{2}$ and $W_{1}+W_{2}$ in case $p \equiv 2(\bmod 3)$, and if $p \equiv 1(\bmod 3)$, we also have $L_{1}+W_{1}, L_{1}+W_{2}, L_{2}+W_{1}$, $L_{2}+W_{2}, L_{1}+W_{1}+W_{2}$ and $L_{2}+W_{1}+W_{2}$.

We would now like to keep only those subspaces which give rise to pairwise nonisomorphic semisymmetric covers. To find all $H$-admissible $p$-elementary abelian regular covering projections which are not $G$-admissible we need to indetify those $\langle R, S\rangle$-invariant subspaces, which are not invariant for the matrix

$$
T=\left(\tau^{\#}\right)^{t}=\tau^{\#^{*}}=\left(\begin{array}{cccccccc}
-1 & 0 & 0 & 0 & 0 & 0 & 0 & 0 \\
-1 & 0 & 0 & 0 & -1 & 0 & 0 & 0 \\
0 & 0 & 0 & -1 & 0 & 0 & 0 & 0 \\
0 & 0 & -1 & 0 & 0 & 0 & 0 & 0 \\
1 & -1 & 0 & 0 & 0 & 0 & 0 & 0 \\
1 & 0 & 0 & 0 & 0 & 0 & 0 & -1 \\
0 & 0 & 0 & 0 & 0 & 0 & -1 & 0 \\
-1 & 0 & 0 & 0 & 0 & -1 & 0 & 0
\end{array}\right)
$$


Direct computation shows that $T$ preserves the subspaces $L_{1}, L_{2}$ (and hence $K_{0}$ ), and interchanges $W_{1}$ and $W_{2}$. Consequently, $W_{1}+W_{2}, L_{1}+W_{1}+W_{2}$ and $L_{2}+W_{1}+W_{2}$ are also $T$ invariant while $K_{0}+W_{2}=T\left(K_{0}+W_{1}\right), L_{1}+W_{2}=T\left(L_{1}+W_{1}\right)$ and $L_{2}+W_{2}=T\left(L_{2}+W_{1}\right)$. The list of semisymmetric covers thus reduces to those arising from the subspaces $W_{1}$ and $K_{0}+W_{1}$ in case $p \equiv 2(\bmod 3)$, and if $p \equiv 1(\bmod 3)$, then we also have $L_{1}+W_{1}$ and $L_{2}+W_{1}$.

In order to further reduce the list of these covering projections to the list of pairwise nonisomorphic ones we proceed to find the orbits of the action of (Aut $\mathcal{H})^{\#^{*}}$ on the above invariant subspaces. To this end we need to compute the matrix

$$
O=\left(\omega^{\#}\right)^{t}=\omega^{\# *}=\left(\begin{array}{cccccccc}
-1 & 0 & -1 & 1 & -1 & -1 & 1 & -1 \\
-1 & 1 & 0 & 1 & 0 & 0 & 1 & 0 \\
-1 & 0 & 0 & 1 & -1 & 0 & 0 & 0 \\
0 & 0 & 0 & 0 & -1 & 0 & 0 & -1 \\
1 & 0 & 0 & -1 & 0 & 1 & -1 & 0 \\
1 & 0 & 0 & -1 & 1 & 0 & -1 & 0 \\
0 & 0 & -1 & 0 & 0 & -1 & 0 & 0 \\
-1 & 0 & 0 & 0 & 0 & -1 & 1 & 0
\end{array}\right)
$$

One can check directly that none of the above spaces is invariant under the action of $O$. Consequently, $H$ is the maximal group that lifts along any of the respective semisymmetric covering projections. Indeed, if there were an automorphism $g \in$ Aut $\mathcal{H}, g \notin H$ which had a lift, then $\langle H, g\rangle$ would be semisymmetric and hence contained in the maximal semisymmetric subgroup $M \cong \operatorname{PSL}(2,7)$. Since $H$ is maximal in $M$, the group $M$ would lift, a contradiction since $\omega \in M$. This also shows that the above semisymmetric covering projections are pairwise nonisomorphic. Indeed, let $U_{1}$ and $U_{2}$ be two of the above $\langle R, S\rangle$-invariant subspaces such that for some $g \in \operatorname{Aut} \mathcal{H}$ we have $g^{\#^{*}}\left(U_{1}\right)=U_{2}$ (actually, $\left\{U_{1}, U_{2}\right\}=\left\{L_{1}+W_{1}, L_{2}+W_{1}\right\}$ because this is the only pair having the same dimension). Then, for each $h \in H$ we have $\left(g^{-1}\right)^{\#^{*}} h^{\#^{*}} g^{\#^{*}}\left(U_{1}\right)=U_{1}$. So $g^{-1} h g$ has a lift. As $H$ is the maximal group that lifts, we have $g^{-1} h g \in H$. Thus, $g$ belongs to the normalizer $G=\langle H, \tau\rangle$. Clearly, $g \notin H$. Consider $\tau^{-1} g$. Since $H$ is of index 2 in $G$ we have $\tau^{-1} g \in H$. Consequently, $\left(\tau^{-1}\right)^{\#^{*}} g^{\#^{*}}\left(U_{1}\right)=U_{1}$ and hence $\tau^{\#^{*}}\left(U_{1}\right)=U_{2}$, implying $\tau^{\#^{*}}\left\{U_{1}, U_{2}\right\}=\left\{U_{1}, U_{2}\right\}$. On the other hand, $\tau^{\#^{*}}\left\{U_{1}, U_{2}\right\}=\tau^{\#^{*}}\left\{L_{1}+W_{1}, L_{2}+W_{1}\right\}=$ $\left\{L_{1}+W_{2}, L_{2}+W_{2}\right\} \neq\left\{U_{1}, U_{2}\right\}$, a contradiction.

So if $p \equiv 1(\bmod 7)$, then the only connected, pairwise nonisomorphic, semisymmetric $p$-elementary abelian regular covering projections onto the Heawood graph arise from the subspaces $W_{1}$ and $K_{0}+W_{1}$ when $p \equiv 2(\bmod 3)$, and if $p \equiv 1(\bmod 3)$, then we also have projections arising from $L_{1}+W_{1}$ and $L_{2}+W_{1}$. This concludes the analysis of case (i).

Cases (ii)-(v) can be carried out analogously, as well as finding all arc-transitive coverings. The results of the semisymmetric case are recollected in Theorem 7.1. (Note that the basis for $W_{1}$ was conveniently changed in order not to involve $\xi$ but, rather, one of the elements $\alpha_{1}, \alpha_{2}$ which are the roots of the polynomial $x^{2}+x+2$.)

Theorem 7.1 Let $p$ be a prime, let $\mathcal{H}$ be the Heawood graph, and let $\zeta, a_{0}, \ldots, a_{6}$ and $a_{7}$ have the same meaning as at the beginning of this subsection. Then: 
If $p \equiv 3,5$, or $6(\bmod 7)$, then there are no connected semisymmetric p-elementary abelian regular covering projections onto $\mathcal{H}$;

If $p \equiv 0,1,2$ or $4(\bmod 7)$, then the isomorphism classes of connected semisymmetric p-elementary abelian regular covering projections onto $\mathcal{H}$ are given in the following table:

Semisymmetric elementary abelian regular covering projections of the Heawood graph.

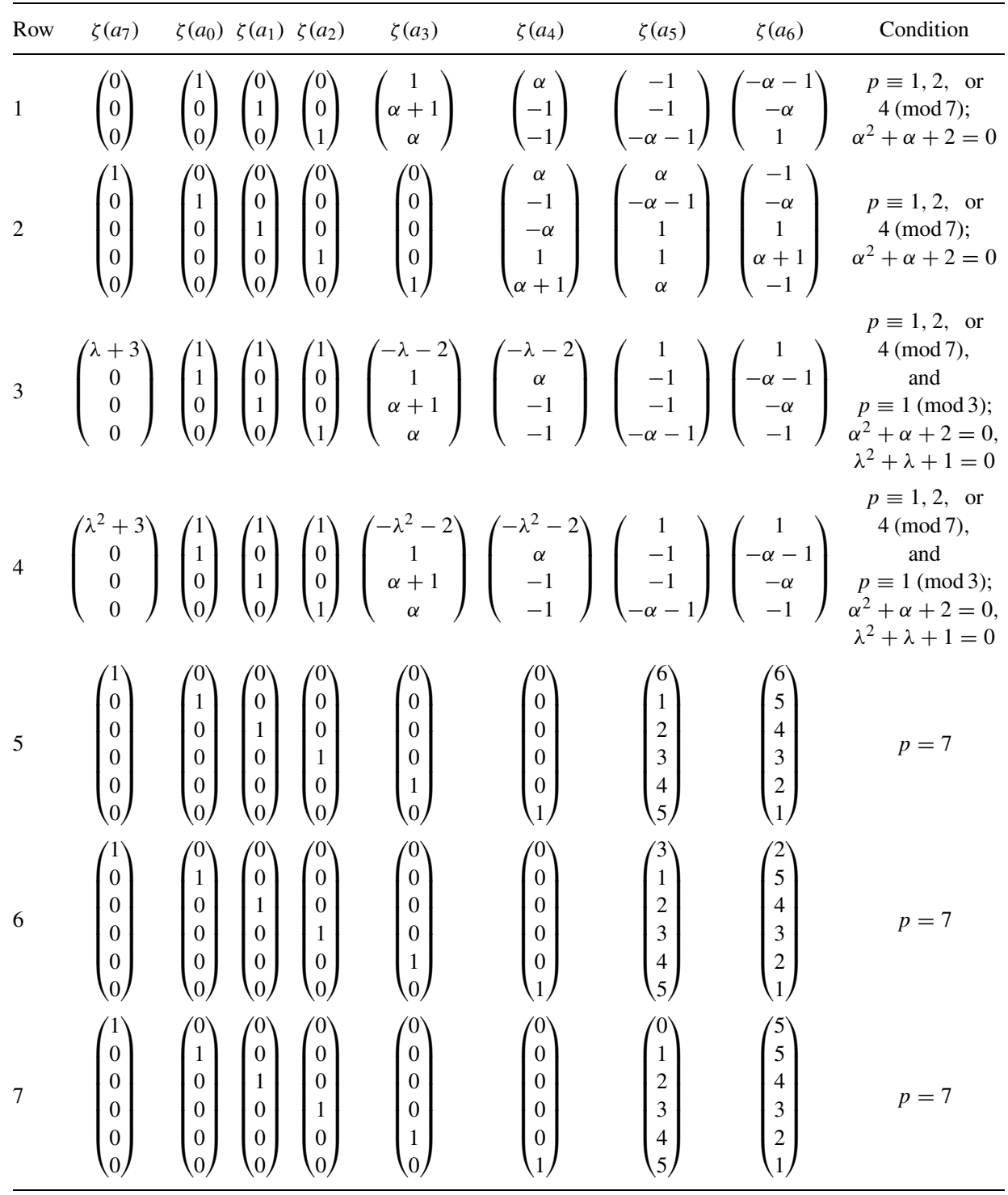




\section{Added in proof}

Recently, a number of papers dealing with the problem of lifting automor-phisms along elementary abelian regular covering projections have appeared or have been submitted for publication [3, 6-8, 9, 16, 17, 21]. In particular, an algorithm which differs from the one presented in this paper appeared in [6]. We also point out that fast randomized algorithms for computing invari-ant subspaces of matrix groups are available, for example Meataxe $[13,19]$, which are also computer implemented (for reasonably small primes) [2, 20]. As for most recent work on semisymmetric graphs see $[3,4,17,26]$.

\section{References}

1. N.L. Biggs, "Homological coverings of graphs," J. London Math. Soc. 30 (1984), 1-14.

2. W. Bosma, C. Cannon, and C. Playoust, "The MAGMA algebra system I: The user language," J. Symbolic Comput. 24 (1997), 235--265.

3. M.D.E. Conder, A. Malnič, D. Marušič, T. Pisanski, and P. Potočnik, "The edge- but not vertex-transitive cubic graph on 112 vertices," J. Graph Theory, to appear.

4. M.D.E. Conder, A. Malnič, D. Marušič, and P. Potočnik, "A census of cubic semisymmetric graphs on up to 768 vertices," submitted.

5. D.Ž. Djokovič, “Automorphisms of graphs and coverings," J. Combin. Theory Ser. B 16 (1974), 243--247.

6. S.F. Du, J.H. Kwak, and M.Y. Xu, "Lifting of automorphisms on the elementary abelian regular coverings," Lin. Alg. Appl. 373 (2003), 101--119.

7. S.F. Du, J.H. Kwak, and M.Y. Xu, "On 2-arc transitive covers of complete graphs with covering transformation group $\mathbb{Z}_{p}^{3}$," J. Combin. Theory Ser. B, to appear.

8. R.Q. Feng and J.H. Kwak, "Typical circulant double coverings of a circulant graph,” Discrete Math. 277 (2004), 73-85.

9. Y.Q. Feng and J.H. Kwak, "s-regular cubic graphs as coverings of the complete bipartite graph $K_{3,3}$," J. Graph Theory 45 (2004), 101-112.

10. C.D. Godsil and G. Royle, Algebraic Graph Theory, Springer-Verlag, New York, 2001.

11. J.L. Gross and T.W. Tucker, Topological Graph Theory, Wiley-Interscience, New York, 1987.

12. M. Hofmeister, "Graph covering projections arising from finite vector spaces over finite fields," Discrete Math. 143 (1995), 87-97.

13. D.F. Holt and S. Rees, "Testing modules for irreducibility," J. Austral. Math. Soc. Ser. A 57 (1994), 1-16.

14. N. Jacobson, Lectures in Abstract Algebra, II. Linear Algebra, Springer-Verlag, New York, 1953.

15. R. Lidl and H. Niederreiter, Finite Fields in Encyclopedia of Mathemaics and Applications, Cambridge Univ. Press, 1984

16. A. Malnič, D. Marušič, and P. Potočnik, "On cubic graphs admitting an edge-transitive solvable group," J. Alg. Combin. 20 (2004), 155-169.

17. A. Malnič, D. Marušič, P. Potočnik, and C.Q. Wang, "An infinite family of cubic edge- but not vertex-transitive graphs," Discrete Math. 280 (2004), 133-148.

18. A. Malnič, R. Nedela, and M. Škoviera, "Lifting graph automorphisms by voltage assignments," Europ. J. Combin. 21 (2000), 927-947.

19. P.M. Neumann and C.E. Praeger, "Cyclic matrices and the Meataxe," in Groups and computation, III (Columbus, OH, 1999), 291--300, Ohio State Univ. Math. Res. Inst. Publ., 8, de Gruyter, Berlin, 2001.

20. M. Schönert et al., GAP_-Groups, Algorithms, and Programming, Lehrstuhl D fur Mathematik, RWTH, Aachen, 1994.

21. D.B. Surowski and C.W. Schroeder, "Homological methods in algebraic map theory," Europ. J. Combin. 24 (2003), 1003-1044.

22. J. Širáň, "Coverings of graphs and maps, orthogonality, and eigenvectors," J. Alg. Combin. 14 (2001), 5772. 
23. M. Škoviera, "A contribution to the theory of voltage graphs," Discrete Math. 61 (1986), 281-292.

24. A. Venkatesh, Covers in imprimitively symmetric graphs, Honours dissertation, Department of Mathematics, University of Western Australia, 1997.

25. A. Venkatesh, "Graph coverings and group liftings," manuscript.

26. S. Wilson, “A worthy family of semisymmetric graphs,” Discrete Math. 271 (2003), 283-294. 